\title{
Implications of the directivity of railway noise sources for their quantification using conventional beamforming
}

\author{
Jin Zhang, Giacomo Squicciarini, David J. Thompson \\ Institute of Sound and Vibration Research, University of Southampton SO17 1BJ, United \\ Kingdom
}

\begin{abstract}
This paper investigates the effect of the directivity of railway noise sources on the results of an identification procedure based on beamforming using a microphone array. Usually when performing pass-by noise tests, a single-microphone noise spectrum is obtained for a time window corresponding to the length of the whole train, or of a single vehicle. In this context, a source quantification algorithm should be able to evaluate the contribution of each noise source over this time window. However, different railway noise sources have different directivities, and it is shown that these need to be taken into account to achieve accurate source quantification. By making use of monopoles, dipoles and quadrupoles, it is shown that a different compensation is needed according to the directivity. For the particular case of the noise radiated by the rail, this has a complex directivity pattern that is only partially captured by a microphone array. It is demonstrated that the overestimation of the wheel contribution found in previous research may be attributed to a misinterpretation of part of the rail contribution from the beamforming map.
\end{abstract}

Keywords: Railway noise, Directivity, Beamforming, Source quantification

Email addresses: Jin.Zhang@soton.ac.uk (Jin Zhang), G.Squicciarini@soton.ac.uk (Giacomo Squicciarini), djt@isvr.soton.ac.uk (David J. Thompson)

Preprint submitted to Journal of ${ }^{A} T_{E} X$ Templates

June 17, 2019 


\section{Introduction}

When a train is running on a straight track, there are three important groups of noise sources: rolling noise excited by surface roughness of the rail and wheel, traction and auxiliary noise, and aerodynamic noise [1, 2]. Specifically, the

5 aerodynamic noise becomes significant when the speed of the train is above about $300 \mathrm{~km} / \mathrm{h}[3$, whereas at lower speeds, the rolling noise plays an important role and is the dominant source for most speeds below $300 \mathrm{~km} / \mathrm{h}$ [1]. The traction noise sources, such as motors and fans, are also important contributors at lower speeds [1].

Aerodynamic noise is generated by air flow over the train components such as the pantograph, the bogies and various gaps and cavities on the train body [3. In general, the aerodynamic noise sources can be characterized as dipoletype and quadrupole-type sources. The dipole ones represent the noise radiated from the flow over cylinders and other bluff bodies 4]; the quadrupole-type sources represent the noise produced by free turbulence [5].

The rolling noise can further be decomposed into wheel and track contributions. Experiments conducted by Thompson and Dittrich [6] showed that the wheel radiation, although characterised by complex directivity patterns, tends to be approximately omnidirectional for radial vibration modes and to behave more similarly to a dipole for axial vibration modes. These approximate directivities for the wheel are used in the TWINS model to predict the wheel noise [7, 8. The rail, on the other hand, has a more complicated radiation pattern. In Ref. [2, a vibro-acoustic rail model was established, the results from which showed that the structural waves travelling in the rail form an extended source of noise radiation. Moreover, the distribution of the sound intensity in the horizontal plane consists of sound propagating at a specific angle, which depends on the ratio between the structural and acoustic wavenumbers [2. This feature was recently adopted to quantify the rail noise contribution by applying a wavenumber filter to the signals from a line array of microphones [9, 10.

Microphone arrays using the beamforming technique are an effective means 
to achieve noise source identification in a wide range of applications [11-17. Beamforming has mainly been introduced for identifying aeroacoustic noise sources 13, 14; however it has also been proposed for isolating the rolling noise contributions [15-17. By suitable processing, the beamforming provides 35 a visualized noise map in which the sources can be found and quantified.

For application to a moving train, it is necessary to 'steer' the array focusing towards a certain point on the train surface and to 'track' this point as it moves along with the train for a certain time. The tracking window should not be excessively long to avoid combining contributions from too many sources.

40 Moreover, the preference for utilizing a short time window for railway measurements has also been explained in Ref. [17, which demonstrates that the selectivity of the array is poor when the tracking point is not in front of the array. Commonly, the array is placed 5 to $10 \mathrm{~m}$ away from the rail. For a high speed train running at $300 \mathrm{~km} / \mathrm{h}$, the valid time window based on a tracking 45 angle smaller than $\pm 20^{\circ}$ is less than $0.1 \mathrm{~s}$, which is quite short compared with the ones used during aircraft fly-over measurements 18 . On a typical beamforming map (see [17, 19] for example), the noise radiated from the wheels can often be seen as well as the aerodynamic noise. The rail contribution is usually not clearly observed.

50 Ideally, the interpretation of the acoustic maps also involves the quantification of the noise sources shown on the map. Several attempts have been made over the years to achieve source quantification from the beamforming results [14, 17, 20,22]. However, an important limitation is that the beamforming technique is often established on the assumption that the noise sources are uncorrelated monopoles. This assumption is in contradiction with the characteristics of the aerodynamic and rolling noise sources on a train. For instance, the absence of the majority of the rail contribution in beamforming maps is likely to be due to the strong directionality of the sound radiated by the rail [2].

In order to discuss the influence of the directivities of railway noise sources on 60 the beamforming results, various examples are considered in this paper. Firstly, a quantification approach designed for train pass-by measurements is introduced 
in Section 2. It makes use of a 72 microphone virtual array and has the capability to quantify the spectrum of a moving monopole accurately by applying a compensation factor. Based on this approach, sources with simple directivities, such as dipoles and quadrupoles, are studied in Section 3. Such sources are representative of aerodynamic phenomena as well as of wheel noise. In order to quantify these sources correctly, additional compensations determined based on the source directivities are introduced. In Section 4 the noise radiated by the rail is analysed numerically and assessed with the quantification approach outlined in Section 2, The rail vibration and its sound radiation are calculated based on a similar approach to Ref. 2] but with the rail excited by a harmonic moving load. Using the simulations, it is investigated to what extent the rail directivity influences the beamforming results. The influence of the noise generated by the rail vibration in the vicinity of the forcing point is given particular consideration.

\section{Beamforming for moving sources and the quantification approach}

\subsection{Beamforming for moving sources}

Frequently, the beamforming techniques for moving sources, such as the ones applied to aircraft fly-over measurements and vehicle pass-by measurements, are based on the assumption that all the sources are uncorrelated monopoles [1517, 22. The beamforming algorithm adopted in this study also makes use of this assumption and relies on applying delays on the output of each microphone channel to give estimations of the potential locations of the sources.

In far field, the sound pressure of a moving monopole recorded by the $m$-th microphone of an array with $N_{M}$ microphones is given by [23, 24]

$$
p_{m}(t)=\frac{Q(\tau)}{4 \pi r_{m}(\tau) D^{2}\left(\mathbf{r}_{m}\right)}, \quad \tau=t-\frac{r_{m}(\tau)}{c_{0}}
$$

in which $r_{m}$ is the magnitude of the distance vector $\mathbf{r}_{m}$ from the source to the $m$-th microphone and $Q(\tau)$ denotes the derivative of the mass flow of the source. 
This is sometimes expressed as $q_{s}^{\prime}(\tau)$ with $q_{s}$ representing the mass flow, which in this case is the product between air density and volume velocity. The term $D(\cdot)$ in Eq. (1) denotes the Doppler coefficient which is given by

$$
D\left(\mathbf{r}_{m}\right)=\left|1-\frac{\mathbf{V} \cdot \mathbf{r}_{m}(\tau)}{c_{0} r_{m}(\tau)}\right|
$$

where $\mathbf{V}$ denotes the vector of the velocity of the source in Eq. (1), $c_{0}$ is the speed of sound. Eq. 1 neglects the near-field terms produced by the source motion [23. The amplitude difference with and without the near-field terms is less than $0.1 \mathrm{~dB}$ when the receiver is at $7.5 \mathrm{~m}$ from the line of motion of the source.

Essentially, the beamforming for moving sources, similar to its implementation for static sources, is achieved by the summation of the array-microphone signals after they have been rescaled and delayed in terms of a focusing point $j$. This is given by

$$
\tilde{q}_{j}(t)=\sum_{m=1}^{N_{M}} h_{j m} p_{m}\left(t+\frac{r_{j m}(t)}{c_{0}}\right)
$$
$\mathrm{A}$

Commonly, for railway pass-by measurements, a planar array is used with the array placed parallel to the surface of the train. The scan plane consists of 


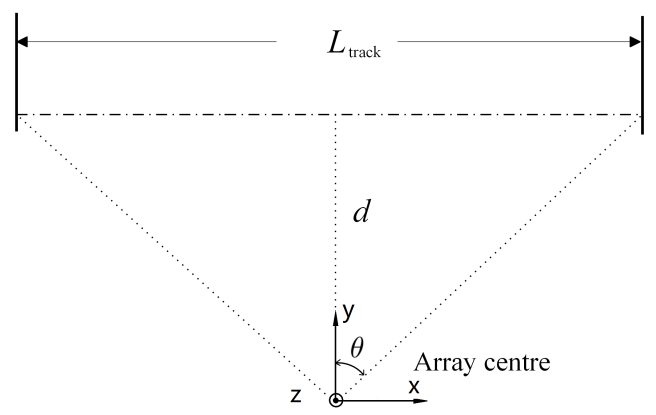

Figure 1: A schematic plan view to illustrate the coordinate system and the tracking angle $\theta$.

For the simulations included in this paper, the 72 microphone array shown in Fig. 2 is used. This array has a $3-\mathrm{dB}$ resolution (the diameter of the main lobe $3 \mathrm{~dB}$ below the peak) 25 . of $2.2 \mathrm{~m}$ at $500 \mathrm{~Hz}$ while at $5 \mathrm{kHz}$ the maximum side lobe [26] is around $12 \mathrm{~dB}$ below the peak for a single point source. A reference microphone close to the centre is also identified in the figure. For the following numerical simulations presented, a sampling frequency of $10 \mathrm{kHz}$ has been used to generate the signals in time domain. 


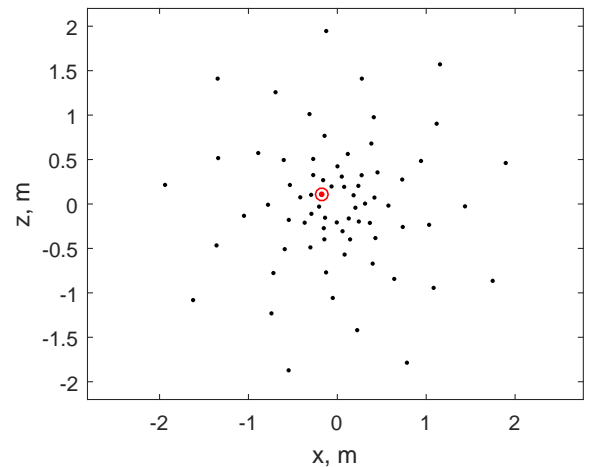

Figure 2: Array configuration used for simulations. The point marked by a circle denotes the location of the reference microphone.

\subsection{Quantification approach}

The quantification approach adopted in this paper is based on the Source Power Integration (SPI) method 27. It can be divided into the following steps:

(1) A frequency-dependent compensation factor is first obtained numerically to relate the beamforming output to the SPL contribution at a reference microphone (more details are given below.)

(2) The acoustic maps for the moving sources are calculated by means of a beamforming procedure.

(3) The various sources on the map are identified and enclosed in a certain predefined area centred at the peak of the main lobe of the source.

(4) The acoustic map is integrated over these areas to give a frequency-dependent result for each of the sources identified on the map.

(5) The compensation factor is applied to the integrated beamforming result to give the spectrum of each source.

To calculate the compensation factor, a numeral simulation with a moving monopole is performed. In this simulation the spectrum at a single reference microphone, $L_{\mathrm{ref}}$, is first calculated when the source is moving over a predefined length. In railway measurements, it is convenient to set this length to correspond 
to a single vehicle of the train (usually between 18 and $26 \mathrm{~m}$ ) as this would give the result in the form $L_{\text {eq, }} T_{p}$ defined in the ISO 3095 standard [28]. Subsequently, steps (2) to (5) of the quantification technique are applied to this monopole source to obtain the spectrum of the source $L_{\text {map }}$ via the map. To obtain this quantity, the tracking window in the dedopplerization procedure must be the same as the one that will be adopted in the actual test. This is generally shorter than the one used to calculate $L_{\text {ref. }}$. The compensation factor is calculated as the decibel difference between $L_{\text {ref }}$ and $L_{\text {map }}$ in each frequency band.

A numerical example of the result of the compensation is given in Fig. 3 . A monopole moving at $300 \mathrm{~km} / \mathrm{h}$ from left to right is adopted. It starts at $[-13,7.5,0] \mathrm{m}$. A reference length of $26 \mathrm{~m}$ is applied here to obtain the spectrum at the reference microphone (see Fig. 2). The array is located at $7.5 \mathrm{~m}$ from the scan plane containing the source. The acoustic maps are calculated by using a tracking window of length $2.65 \mathrm{~m}$ which corresponds to $\theta$ of $10^{\circ}$. An example at $1 \mathrm{kHz}$ is given in Fig. 3(a). On the map, the identified source is found at $[0,7.5,0] \mathrm{m}$. The integration area centred at the peak of the map is highlighted with white lines. In Fig. 3(b), the spectra of the compensation factors obtained using different sizes of integration area from $2.4 \mathrm{~m} \times 2.4 \mathrm{~m}$ to $4.8 \mathrm{~m} \times 4.8 \mathrm{~m}$ are shown. Differences between these results are seen below $800 \mathrm{~Hz}$. The compensation factors are calculated only at the central frequencies of each $1 / 3$ octave band and are used later to compensate the broadband spectra. Moreover, to remove the unwanted contributions from the side lobes, a threshold level is applied. Those beamforming outputs below the level of the peak minus the threshold level are regarded as background noise and will not be taken into account in the integration. The implementation of the threshold for beamforming quantification has been validated in previous research [29]. In this paper, a threshold level of $12 \mathrm{~dB}$ is used. In Fig. 3(a), the maximum level is $51.3 \mathrm{~dB}$. Hence, the outputs below $39.3 \mathrm{~dB}$ are neglected during the integration.

To validate this quantification approach, a simulation similar to the one presented in Fig. 3 is made next. Herein, a monopole with a speed of 300 


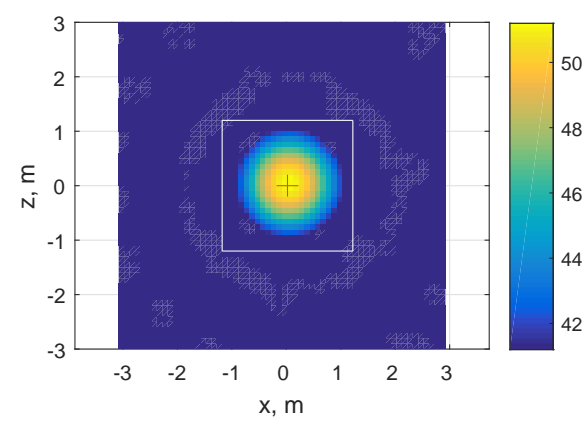

(a)

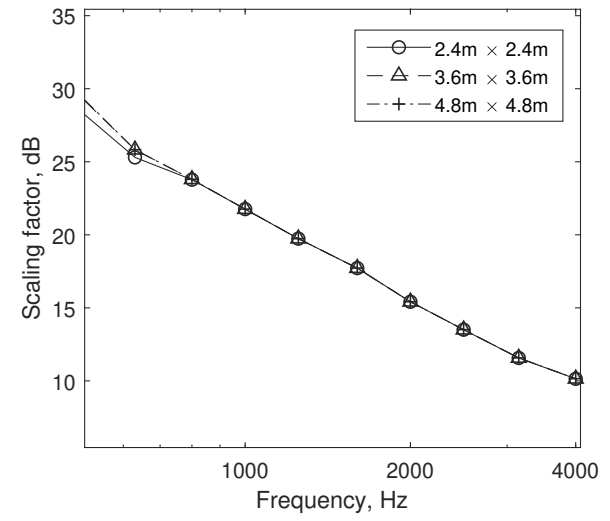

(b)

Figure 3: (a) An example of an acoustic map of a moving monopole source with a speed of $300 \mathrm{~km} / \mathrm{h}$ moving from $[-13,7.5,0] \mathrm{m}$ to $[13,7.5,0] \mathrm{m}$. The red cross denotes the peak on the map. The white rectangle denotes the integration area. The dynamic range of the map is 10 dB. (b) Compensation spectra obtained using different integration areas.

$\mathrm{km} / \mathrm{h}$ is used which is emitting random noise generated by using the embedded function 'randn' in MATLAB, which can be regarded as an approximation to white noise. The estimated source spectra and the spectrum obtained from the reference microphone are given in Fig. 4. In this figure, source estimations are obtained by applying the compensation spectra on the integrated beamforming results using the corresponding integration areas. The quantification results of the acoustic maps are within less than $0.3 \mathrm{~dB}$ of each other for different sizes of the integration area. Moreover, all these estimated spectra show quite good agreement with the spectrum of the reference microphone. However, deviations of up to $1.5 \mathrm{~dB}$ are found.

There are two possible reasons for these differences. First, a uniform factor is used to compensate each $1 / 3$ octave band, whereas Fig. 3(b) indicates that it should be frequency dependent. Nevertheless a maximum deviation of \pm 1 $\mathrm{dB}$ would be expected from this simplification. Second, due to the Doppler effect, the source signal will be redistributed into the adjacent $1 / 3$ octave bands, 


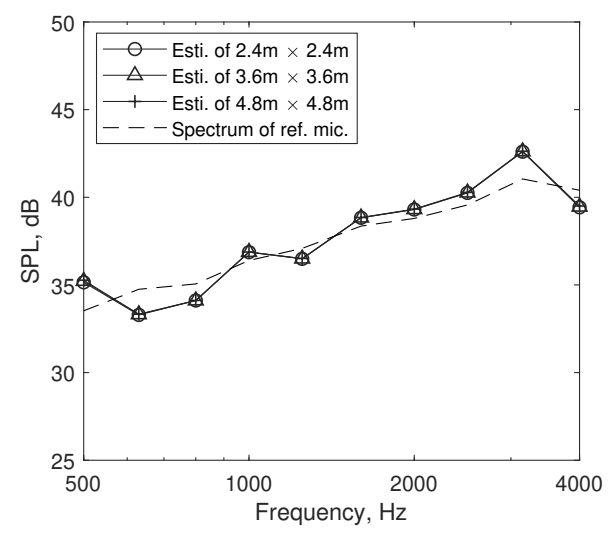

Figure 4: Spectrum of the reference microphone signals (dash-dot line) and estimation results (lines with markers).

especially for components that are close to the band limits. This spectrum redistribution will be included in $L_{\text {ref }}$ but not in the dedopplerised signals.

200 unit source strength used in this figure, which has a frequency of $1 \mathrm{kHz}$, moves from $[-13,7.5,0] \mathrm{m}$ to $[13,7.5,0] \mathrm{m}$ at different speeds. A single microphone is placed at $[0,0,0] \mathrm{m}$. The redistribution becomes greater as the source velocity is increased. At $50 \mathrm{~km} / \mathrm{h}$, the received signal still remains within the $1 / 3$ octave made using noise generated in the same way as that used in Fig. 4. Three speeds, 50, 180 and $300 \mathrm{~km} / \mathrm{h}$, are used to demonstrate the influence of the Doppler effect on the reference spectra and on the quantification results. The results are presented in Fig. 6 based on 50 simulations at each given speed. The thin band. When the speed is $180 \mathrm{~km} / \mathrm{h}$, it extends to the lower limit of the band and to around $20 \mathrm{~Hz}$ above the upper limit. At $300 \mathrm{~km} / \mathrm{h}$, the redistribution into the adjacent $1 / 3$ octave bands becomes significant; the spectrum extends from $830 \mathrm{~Hz}$ to $1270 \mathrm{~Hz}$, which is beyond the band limits of $891 \mathrm{~Hz}$ to $1120 \mathrm{~Hz}$.

To show the effect of speed on the array output, additional simulations are lines are the spectra of the reference microphone and are obtained by using the 


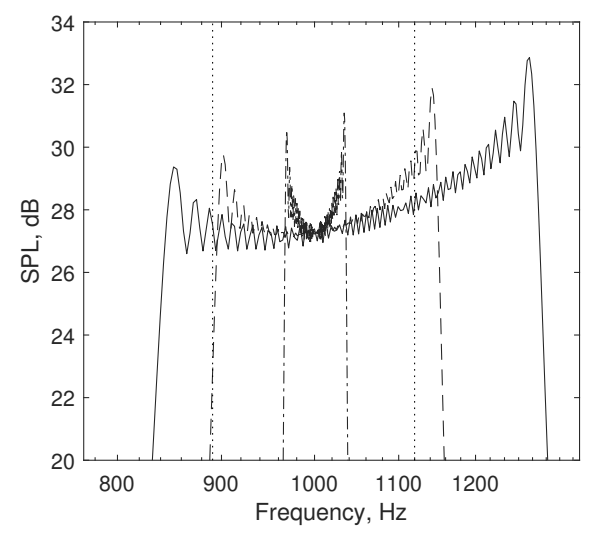

Figure 5: The spectra of a monopole pass-by at different speeds: '-.', $50 \mathrm{~km} / \mathrm{h}$; '--', 180 $\mathrm{km} / \mathrm{h} ;{ }^{-}-', 300 \mathrm{~km} / \mathrm{h}$. The sensor is placed at $[0,0,0] \mathrm{m}$. The source with frequency $1 \mathrm{kHz}$ moves from $[-13,7.5,0] \mathrm{m}$ to $[13,7.5,0] \mathrm{m}$. The vertical lines mark the limits of the $1 / 3$ octave band centred at $1 \mathrm{kHz}$.

simulated signals directly, therefore without applying dedopplerisation. The thick lines are the map quantification outcomes. In each case, the solid line shows the averaged levels over the simulations and the dash-dot lines indicate the average values plus/minus the corresponding standard deviations. According to the figures, the quantification approach proposed in this paper can provide a good estimation of the source in a statistical sense. The level deviation between the averaged reference spectra and the averaged map quantification outcomes are within $0.5 \mathrm{~dB}$ at the three speeds. However, the standard deviation is enlarged when the source speed increases. At $50 \mathrm{~km} / \mathrm{h}$, the standard deviation 225 is $0.2 \mathrm{~dB}$ for the reference spectrum and $0.6 \mathrm{~dB}$ for the map quantification. This becomes $0.5 \mathrm{~dB}$ and $1.5 \mathrm{~dB}$ respectively at $300 \mathrm{~km} / \mathrm{h}$. This confirms that the deviations found in Fig. 4 are a consequence of a combination of the source motion as well as the frequency content of the source spectrum. 


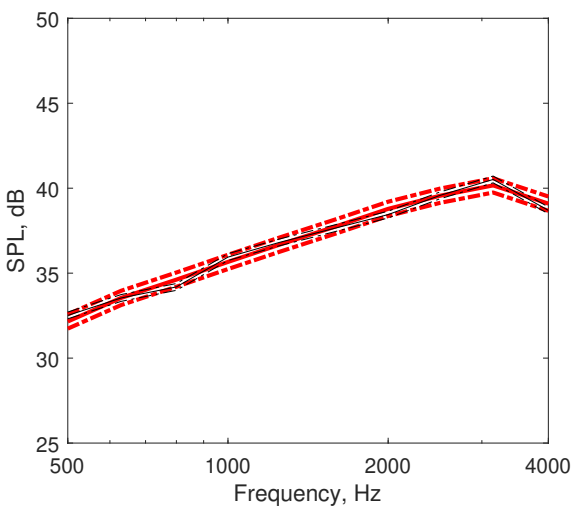

(a)

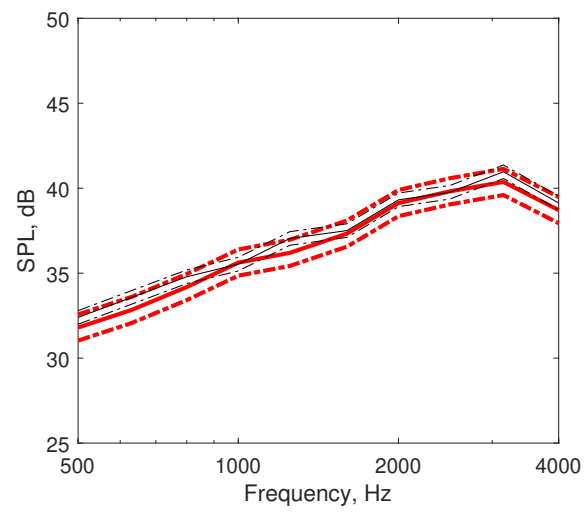

(b)

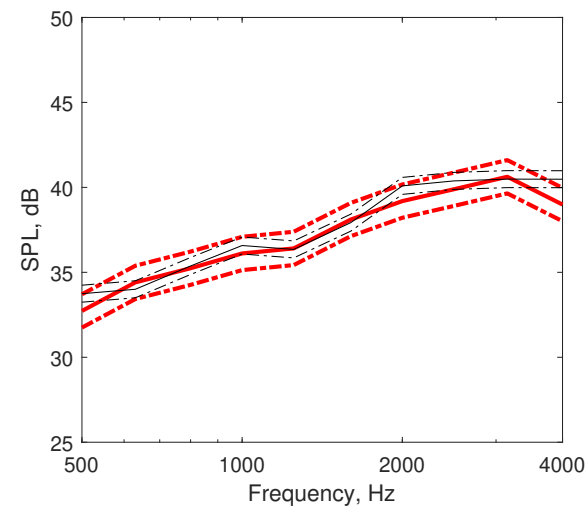

(c)

Figure 6: Statistical results of the reference spectra (thin lines) and the map quantification outcomes (thick lines): (a) at $50 \mathrm{~km} / \mathrm{h}$; (b) at $180 \mathrm{~km} / \mathrm{h}$; (c) at $300 \mathrm{~km} / \mathrm{h}$. The solid lines are the averaged values. The dash-dot lines define the mean $+/$ - one standard deviation. 


\section{Quantification of sources with simple directivity using beamform- ing}

As described previously, the beamforming algorithm relies on the assumption that the source radiates omnidirectionally. However, the monopole assumption is not well suited for many railway noise sources due to their specific directivities and this can lead to mis-interpretation of the acoustic maps.

In this section, numerical simulations are presented for moving dipole and longitudinal quadrupole sources to discuss this phenomenon. These sources are oriented in such a way that the axis corresponding to maximum directivity is perpendicular to the direction of motion and therefore they radiate predominately towards the array. The same parametric settings as used in Section 2 are applied here, such as the reference microphone location, source speed and tracking windows. An integration area of $2.4 \mathrm{~m} \times 2.4 \mathrm{~m}$ is adopted for the quantification procedure. The same random signals are adopted here as used in the previous section.

In far field, the sound pressure produced by these two types of source can be obtained by introducing direction-dependent terms into Eq. (1). The dipole then is given by [23, 30]

$$
p_{m}(t)=\frac{Q(\tau) \cos \phi(\tau)}{4 \pi r_{m}(\tau) D^{2}\left(\mathbf{r}_{m}\right)}
$$

and for quadrupole it is

$$
p_{m}(t)=\frac{Q(\tau) \cos ^{2} \phi(\tau)}{4 \pi r_{m}(\tau) D^{3}\left(\mathbf{r}_{m}\right)}
$$

where the angle $\phi(\tau)$ is given by

$$
\phi(\tau)=\cos ^{-1}\left(\frac{y_{s}(\tau)-y_{m}}{\left|\mathbf{r}_{m}(\tau)\right|}\right)
$$

and $y_{s}(\tau)$ and $y_{m}$ denote the positions of the source and the $m$-th microphone on the $y$-axis. The relationship between $t$ and $\tau$ is the same as that given in Eq. (1). The derivation of Eq. (6) is given in Appendix B. Both Eqs. (5) and (6) neglect the near-field terms. The amplitude differences for these two directional 
sources are less than $0.1 \mathrm{~dB}$ with the near-field terms. The directivity patterns and pressure distribution maps of these two types of source are given in Fig. 7 Specifically, Figs. $7(\mathrm{~d})$ and $7(\mathrm{f})$ show the SPL distributions of the instantaneous acoustic fields generated by the directional sources in a fixed frame when the sources are at $[0,7.5,0] \mathrm{m}$. It can be seen that the directionality is greater for the quadrupole than for the dipole. The results obtained by using a monopole source are also shown in the figure for comparison.

The beamforming responses of the moving dipole and quadrupole are shown in Figs. 8(a) and 8(c) These two maps are nearly identical to the map generated by a moving monopole (Fig. 3(a) . A single bright spot can be seen on each map and the peaks, marked with crosses, are each found at $[0,7.5,0] \mathrm{m}$. Due to the short duration of the tracking window the microphone array is not sensitive to the directivity of the sources.

On the other hand, following railway noise standards [28, the spectrum for a single microphone is obtained by using a signal with a longer duration corresponding at least to the pass-by time of an entire vehicle. Consequently, the single microphone spectrum of a directional source will have a lower level than the one obtained using an omnidirectional source due to the source directivity. Figs. 8(b) and 8(d) give the spectrum at the single microphone as dashed lines. For the dipole, the single microphone spectrum has a deviation which is $1 \mathrm{~dB}$ to $3.5 \mathrm{~dB}$ lower compared to the estimate obtained from the beamforming map, and the difference is on average around $2 \mathrm{~dB}$. The averaged difference increases to around $3 \mathrm{~dB}$ with deviations from $2 \mathrm{~dB}$ to $4 \mathrm{~dB}$ when the quadrupole source is considered. Thus differences between the acoustic map estimations and the single microphone spectra appear and the disagreement increases with increasing source directivity. Additionally, it can be noted that the estimated spectra (solid lines in Figs. 8(b) and 8(d) , obtained through the same quantification approach described above, are almost identical to the estimation for the moving monopole.

To overcome this estimation inaccuracy, an additional compensation $L_{D C}$ 


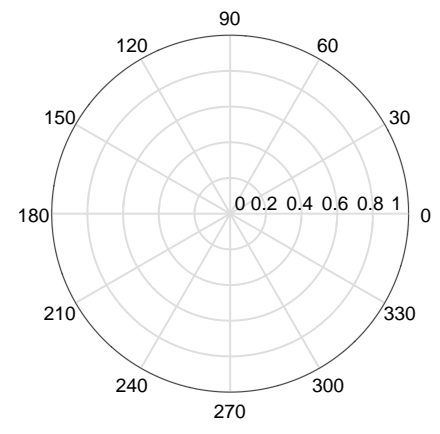

(a)

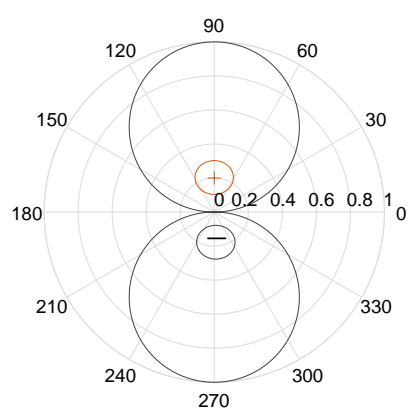

(c)

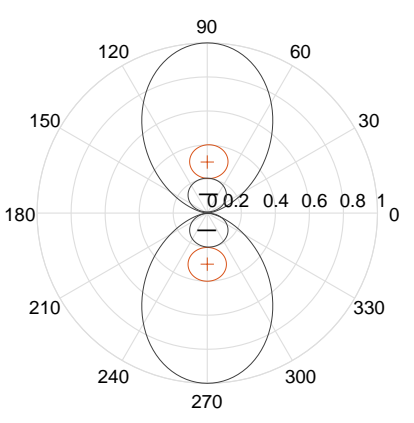

(e)

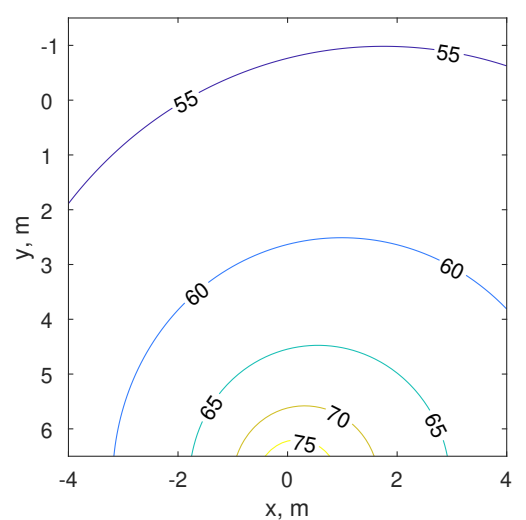

(b)

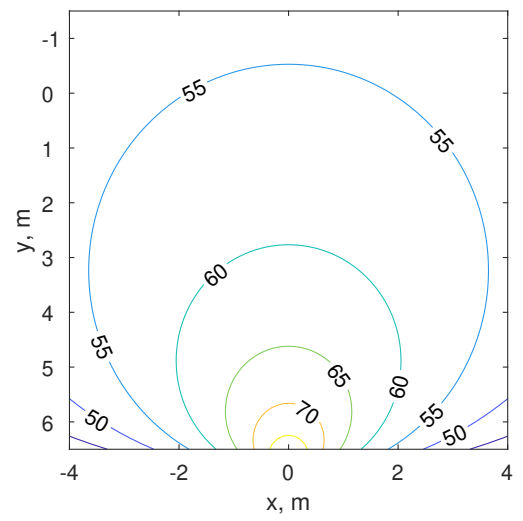

(d)

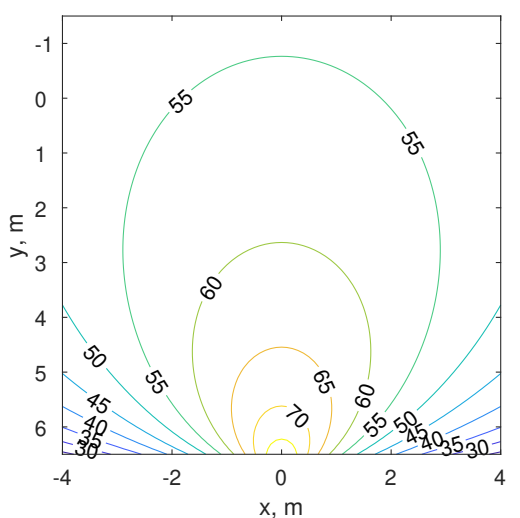

(f)

Figure 7: (a) The directivity of a monopole. (b) The instantaneous SPL distribution map of a moving monopole at $[0,7.5,0] \mathrm{m}$. (c) The directivity of a dipole. (d) The instantaneous SPL distribution map of a moving dipole at $[0,7.5,0] \mathrm{m}$. (e) The directivity of a longitudinal quadrupole. (f) The instantaneous SPL distribution map of a moving longitudinal quadrupole at $[0,7.5,0] \mathrm{m}$. The source moves at a speed of $300 \mathrm{~km} / \mathrm{h}$ from left to right. 


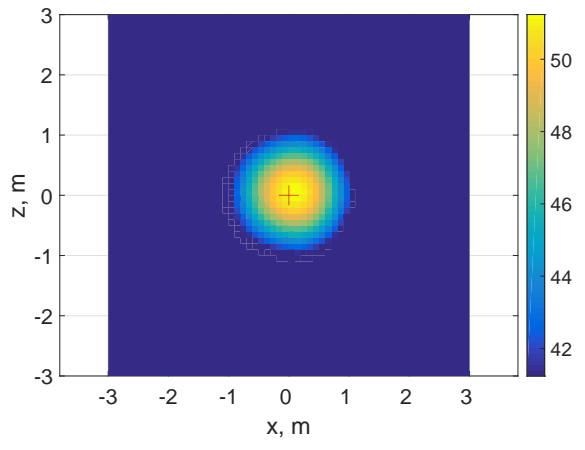

(a)

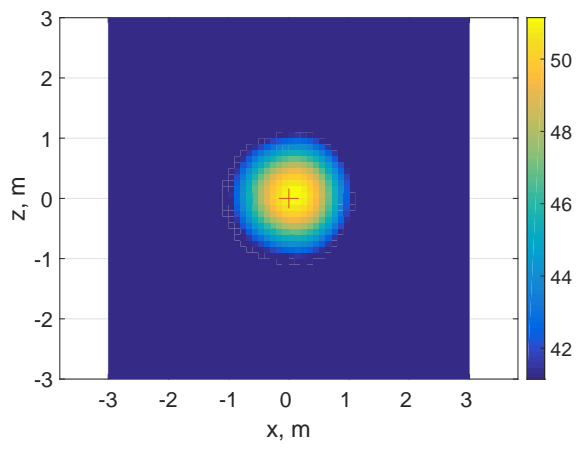

(c)

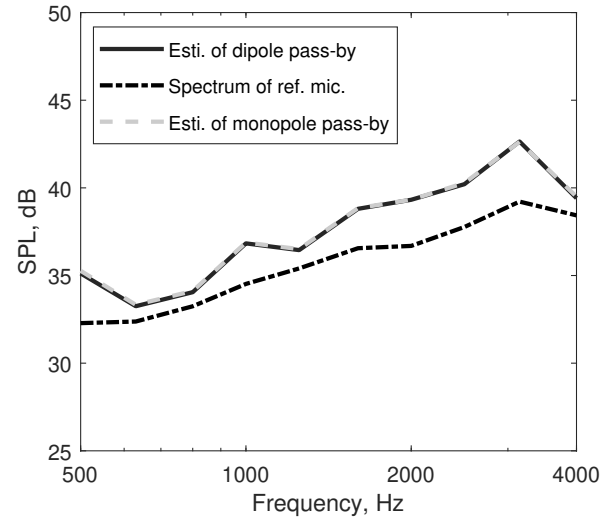

(b)

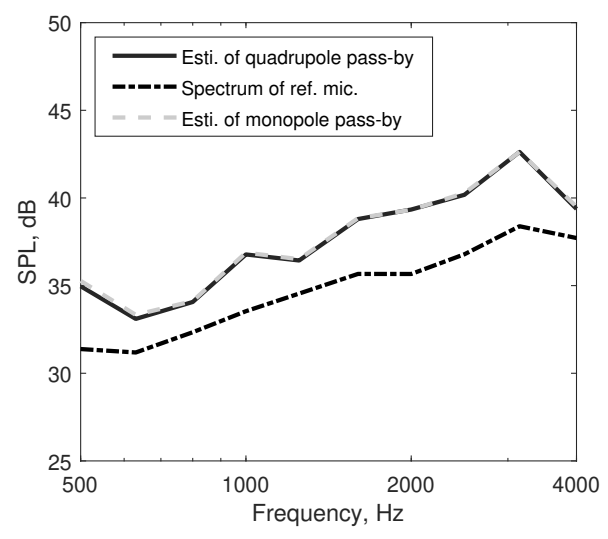

(d)

Figure 8: (a) Acoustic map of a moving dipole at $1 \mathrm{kHz}$; (b) spectrum of the quantification of the acoustic maps of a moving dipole; (c) acoustic map of a moving quadrupole at $1 \mathrm{kHz}$; (d) spectrum of the quantification of the acoustic maps of a moving quadrupole. In (a) and (c), two directional sources are identified at $[0,7.5,0] \mathrm{m}$. The source moves at $300 \mathrm{~km} / \mathrm{h}$. 
based on the source directivity would be required. This is given by

$$
\begin{aligned}
L_{D C} & =20 \log _{10}\left\{\int_{0}^{T}\left|\tilde{p}_{\text {mono }}\right| \mathrm{d} \tau / \int_{0}^{T}\left|\tilde{p}_{\text {direct }}\right| \mathrm{d} \tau\right\} \\
& =20 \log _{10}\left\{\int_{0}^{T} \frac{1}{4 \pi r_{\text {ref }}(\tau) D^{2}\left(\mathbf{r}_{\text {ref }}\right)} \mathrm{d} \tau / \int_{0}^{T} \frac{f[\phi(\tau)]}{4 \pi r_{\text {ref }}(\tau) D^{2}\left(\mathbf{r}_{\text {ref }}\right)} \mathrm{d} \tau\right\}
\end{aligned}
$$

where the subscription ()$_{\text {ref }}$ denotes the microphone used to obtain the reference spectrum and $\phi(\tau)$ denotes the time-dependent angle between a line normal to the track and passing through the array centre and a line from the array centre to the moving source. $\left|\tilde{p}_{\text {mono }}\right|$ denotes the magnitude due to the monopole and $\left|\tilde{p}_{\text {direct }}\right|$ is that due to the directional source. The term $f[\phi(\tau)]$ denotes the directivity of the source. The dipole can be described with $f[\phi(\tau)]=\cos [\phi(\tau)]$ and the longitudinal quadrupole with $f[\phi(\tau)]=\cos ^{2}[\phi(\tau)] / D\left(\mathbf{r}_{\text {ref }}\right)$.

The quantification results obtained with these compensations based on the source directivity are given in Fig. 9. The integration length $T$ applied in Eq. 8 is calculated according to the reference distance of $26 \mathrm{~m}$ used for the simulations presented in Section 2.2. In Fig. 9(a) a rescaled estimate of the dipole is shown as a dot line. A compensation of $2.0 \mathrm{~dB}$ has been applied for the moving dipole. For the moving quadrupole, the rescaling factor is $3.5 \mathrm{~dB}$ according to Eq. (8), and the results for rescaled spectrum is shown in Fig. 9(b) A good match between the single-microphone spectrum and the acoustic map estimation is achieved by implementing the directivity compensation. For the dipole case, the average deviation is reduced to $-0.1 \mathrm{~dB}$ from $2.0 \mathrm{~dB}$ without the compensation. For the longitudinal quadrupole, the averaged deviation is $-0.6 \mathrm{~dB}$ compared with $2.9 \mathrm{~dB}$ without the compensation. Therefore, according to these results, a good quantification of a given acoustic map relies on prior knowledge of the directivity of the source. The remaining differences are associated with the Doppler effect, as seen for the monopole in Fig. 6 .

\section{Rail noise and its beamforming response}

Due to its vibration characteristics, the rail cannot be considered as a compact source and needs to be addressed separately. To understand how its sound 


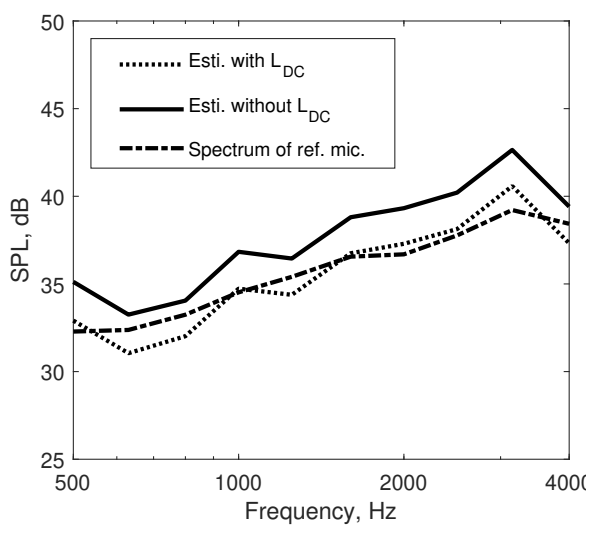

(a)

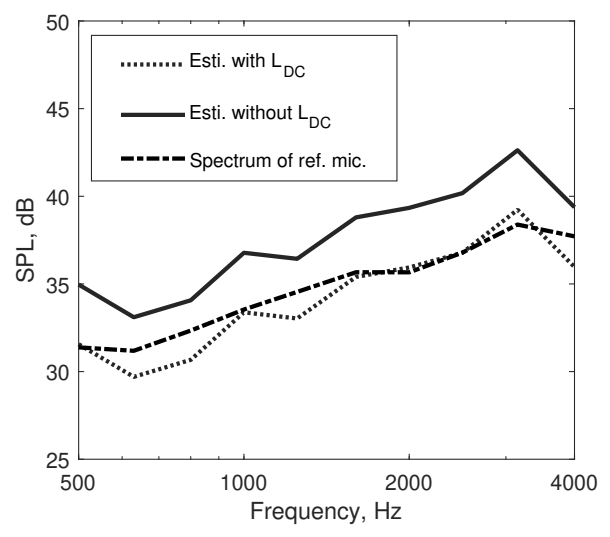

(b)

Figure 9: (a) Spectrum of the quantification of the acoustic maps of a moving dipole; (b) spectrum of the quantification of the acoustic maps of a moving quadrupole. The source moves at $300 \mathrm{~km} / \mathrm{h}$.

radiation influences the beamforming responses, a dynamic model and an acoustic model are first developed. They provide the sound field generated by a harmonic moving load on the rail, which will then be analysed with the same virtual array adopted in Sections 2 and 3 .

\subsection{Rail vibration excited by a moving load}

The rail vibration is modelled using a Timoshenko beam on a continuous two-layer foundation [2]. A schematic of this model is presented in Fig. 10 Only the rail vibration in the vertical direction is considered, although a model

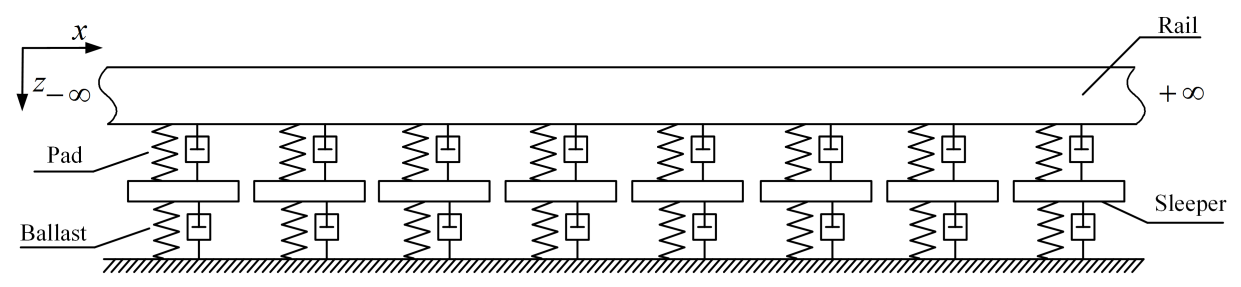

Figure 10: Beam on continuous two-layer foundation

for the lateral direction can be built in an equivalent way. In order to simulate 
the rail radiation when the train is passing in front of the array, a harmonic moving force is applied. The model, therefore, can be described by

$$
\begin{aligned}
& G A \kappa \frac{\partial}{\partial x}\left(\phi-\frac{\partial u}{\partial x}\right)+s u+\rho A \frac{\partial^{2} u}{\partial t^{2}}=F e^{i \omega t} \delta(x-V t) \\
& G A \kappa\left(\phi-\frac{\partial u}{\partial x}\right)-E I \frac{\partial^{2} \phi}{\partial x^{2}}+\rho I \frac{\partial^{2} \phi}{\partial t^{2}}=0
\end{aligned}
$$

where $u$ is the rail displacement, $\phi$ is the rotation of the rail cross-section relative to the undeformed axis, $V$ is the load speed and $s$ is the effective support stiffness per unit length which is given by

$$
s=\frac{s_{p}\left(s_{b}-\omega^{2} m_{s}^{\prime}\right)}{s_{p}+s_{b}-\omega^{2} m_{s}^{\prime}}
$$

where $s_{p}$ is the dynamic stiffness of the rail pad per unit length of track, $s_{b}$ is the dynamic stiffness of the ballast per unit length and $m_{s}^{\prime}$ is the sleeper mass per unit length. The argument of the Dirac function in Eq. 9 describes the motion of the excitation. In the model, damping is introduced by making the Young's modulus and the various stiffnesses complex. For example, $E$ is modified as $E(1+\mathrm{i} \eta)$ where $\eta$ is the loss factor. The parameters used in Eq. (9) and Eq. 10 for the following simulations are listed in Table 1.

A solution to Eqs. (9) and (10) can be found by applying a double Fourier transform from space-time to the wavenumber-frequency domain. The derivation is given in Appendix C and the approach is similar to that presented in [31, 32]. The rail deformation $u$ is given by

$$
\begin{aligned}
& u^{+}(x, t)=-\mathrm{i} F e^{\mathrm{i} \omega t} \sum_{k_{r}^{+}=k_{r, p}^{+}, k_{r, n}^{+}}\left[\frac{p_{n}\left(k_{r}^{+}\right)}{p_{d}\left(k_{r}^{+}\right)} e^{-\mathrm{i} k_{r}^{+}(x-V t)}\right] \\
& x \geqslant V t, \operatorname{Im}\left(k_{r}^{+}\right)<0 \\
& u^{-}(x, t)=\mathrm{i} F e^{\mathrm{i} \omega t} \sum_{k_{r}^{-}=k_{r, p}^{-}, k_{r, n}^{-}}\left[\frac{p_{n}\left(k_{r}^{-}\right)}{p_{d}\left(k_{r}^{-}\right)} e^{-\mathrm{i} k_{r}^{-}(x-V t)}\right] \\
& x \leqslant V t, \operatorname{Im}\left(k_{r}^{-}\right)>0
\end{aligned}
$$

where $F e^{\mathrm{i} \omega t}$ describes the excitation force, and here its amplitude $F$ is set to $3201 ; u^{+}$denotes the rail deformation when $x$ is ahead of the moving excitation 
Table 1: Rail system parameters used in numerical models 2

\begin{tabular}{lll}
\hline & & Vertical \\
\hline Rail bending stiffness & $E I$ & $6.42 \times 10^{6} \mathrm{Nm}^{2}$ \\
Damping loss factor of rail & $\eta$ & 0.02 \\
Rail mass per unit length & $\rho A$ & $60 \mathrm{~kg} / \mathrm{m}$ \\
Rail shear stiffness & $G A$ & $6.17 \times 10^{8} \mathrm{~N}$ \\
Rail shear coefficient & $\kappa$ & 0.4 \\
Rail rotation inertia & $\rho I$ & $0.240 \mathrm{~kg} \cdot \mathrm{m}$ \\
Pad stiffness per unit length & $s_{p}$ & $6.83 \times 10^{8} \mathrm{~N} / \mathrm{m}^{2}$ \\
Pad damping loss factor & $\eta_{p}$ & 0.2 \\
Sleeper mass per unit length & $m_{s}^{\prime}$ & $250 \mathrm{~kg} / \mathrm{m}$ \\
Ballast stiffness per unit length & $s_{b}$ & $3.83 \times 10^{7} \mathrm{~N} / \mathrm{m}^{2}$ \\
Ballast damping loss factor & $\eta_{b}$ & 2.0 \\
\hline
\end{tabular}

force, and $u^{-}$denotes the rail deformation when it is behind the force; $p_{n}$ and $p_{d}$ are both polynomials in terms of the structural wavenumbers $k_{r}$ of the rail (see Appendix C). Specifically, four structural waves that correspond to the four wavenumbers in Eqs. 12 and $(13)$ are included in this model.

325 As explained in Ref. 31, they represent the structural waves of the rail in a reference frame that moves along with the excitation. These are two evanescent waves (corresponding to wavenumbers $k_{r, n}^{+}$and $k_{r, n}^{-}$) which decay rapidly along the rail and two propagating waves (corresponding to wavenumbers $k_{r, p}^{+}$and $\left.k_{r, p}^{-}\right)$which decay slowly along the rail at high frequencies. The decay of the 330 four waves can be characterised by the imaginary parts of their corresponding wavenumbers and, in the railway context, this is often expressed as a decay rate [1] as

$$
\Delta=-8.686 \operatorname{Im}\left(k_{r}\right)
$$


where $\Delta$ is given in $\mathrm{dB} / \mathrm{m}$.

$$
\begin{aligned}
& v^{+}(x, t)=\frac{\partial u^{+}}{\partial t}=F \sum_{k_{r}^{+}}\left[\frac{p_{n}\left(k_{r}^{+}\right)}{p_{d}\left(k_{r}^{+}\right)}\left(\omega+k_{r}^{+} V\right) e^{\mathrm{i}\left(\omega+k_{r}^{+} V\right) t-\mathrm{i} k_{r}^{+} x}\right] \\
& x \geqslant V t, \operatorname{Im}\left(k_{r}^{+}\right)<0 \\
& v^{-}(x, t)=\frac{\partial u^{-}}{\partial t}=-F \sum_{k_{r}^{-}}\left[\frac{p_{n}\left(k_{r}^{-}\right)}{p_{d}\left(k_{r}^{-}\right)}\left(\omega+k_{r}^{+} V\right) e^{\mathrm{i}\left(\omega+k_{r}^{-} V\right) t-\mathrm{i} k_{r}^{-} x}\right] \\
& x \leqslant V t, \operatorname{Im}\left(k_{r}^{-}\right)>0
\end{aligned}
$$

where, similarly to Eqs. 12 and $13, v^{+}$and $v^{-}$denotes the point velocity on both directions.

Here, it is worth mentioning that the damping can be represented by making stiffnesses complex when applying the Fourier transform to solve Eq. 10 involving moving loads. Caution is required if the excitation frequencies approach zero (when the loss factor should be multiplied by $\operatorname{sign}\left(\omega-V k_{r}\right)$ ). But in the current cases, $\omega \gg V k_{r}$ as the wave speeds in the rail are much greater than the moving speed of the excitation, and therefore no significant error is introduced 11. This technique has been successfully adopted in 32. In the scope of this research, the wave speed varies between around $1200 \mathrm{~m} / \mathrm{s}$ to $1900 \mathrm{~m} / \mathrm{s}$ which is much greater than the speed of the load, which is around $83 \mathrm{~m} / \mathrm{s}$.

The influence of the moving excitation on the dynamic behaviour of the 345 rail is shown in Fig. 11. Fig. 11(a), the magnitudes of the rail deformation at the moving excitation point are given. Fig. 11(b) gives the decay rates of the propagating wavenumbers relative to the moving excitation in both directions. Results are also shown for a static excitation. Clearly, the differences caused by the motion of the force are very limited, especially for the point deformation. This can be found in Fig. 11(a), where the three lines representing the negative direction, positive direction and static-excitation cases are almost identical. The vertical lines in each figure indicate the extent of a frequency range named the 'blocked region' [1], within which the decay rates of the propagating wavenumbers are high. When the frequency is above the 'blocked region', the decay rates 
of the propagating wavenumbers drop significantly and the corresponding waves start to propagate.

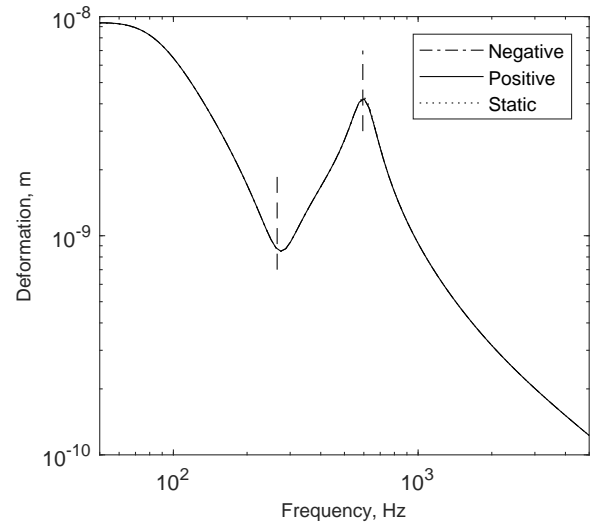

(a)

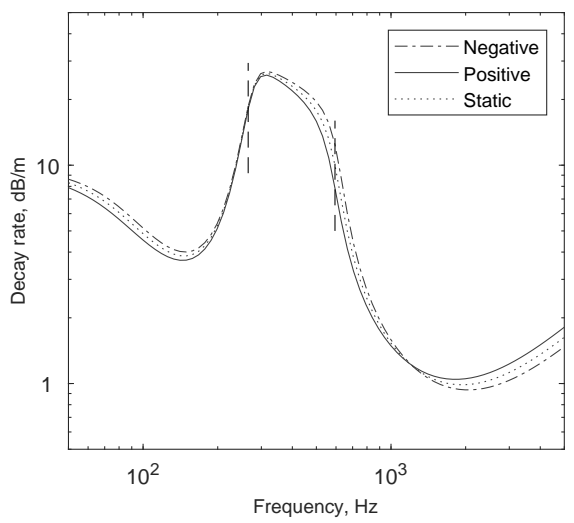

(b)

Figure 11: (a) Point deformations at the excitation point; (b) decay rates of the propagating wavenumbers. The excitation moves at $300 \mathrm{~km} / \mathrm{h}$. Vertical lines indicate the 'blocked region'. In (a), the point deformations obtained with and without source motion are almost identical.

\subsection{Rail noise radiation due to a moving excitation}

According to Ref. [2, 33], the acoustic field generated by the rail can be obtained by representing the rail as a line of coherent elementary sources such as monopoles. The source strength of each monopole on the rail is defined by the corresponding point vibration velocity. Therefore, the sound pressure $p$ observed at $\mathbf{r}_{m}=\left[x_{m}, y_{m}, z_{m}\right]$ can be obtained by integrating the noise radiated from each element of the line source coherently and is given by

$$
p\left(\mathbf{r}_{m}, t\right)=\int_{-\infty}^{\infty} \mathrm{i} \rho_{0} c_{0} k \frac{v(x, \tau)}{4 \pi r_{m}(x)} d x
$$

where $\rho_{0}$ is the density of air, $c_{0}$ is the speed of sound, $k$ denotes the wavenumber in air, $r_{m}$ is the distance from the location $x$ on the rail to $\mathbf{r}_{m}$ and $\tau$ is the emission time, which is related to the receiving time by $\tau=t-r(x) / c_{0}$. Here, an equivalent discrete form of Eq. (17) is used. The rail is decomposed into $N$ monopoles with equal spacing. The distance between two adjacent sources 
is set to be frequency dependent and smaller than a quarter of the acoustic structural wavelength [2]. The discrete form of Eq. (17) at time $t_{j}$ is given by

$$
p\left(\mathbf{r}_{m}, t_{j}\right)=\mathrm{i} \rho_{0} c_{0} k \Delta d\left[\sum_{n=1}^{N} \frac{v\left(x_{n}, \tau_{j n}\right)}{4 \pi r_{m}\left(x_{n}\right)}\right]
$$

where $\Delta d$ is the spacing between two adjacent monopoles, $x_{n}$ denotes the location of the $n$-th monopole on the rail, and $\tau_{j n}$ is the emission time corresponding to $t_{j}$ at the $n$-th monopole. Since the distances from the $m$-th microphone to This indicates that, at each receiving time $t_{j}$, the location of the excitation is different for each element of the track. Thus the point velocity $v$ must be calculated separately for each element at each receiving time $t_{j}$.

Fig. 12 shows the instantaneous pressure maps and the SPL distribution maps for a moving excitation on the rail at $500 \mathrm{~Hz}$ and $1 \mathrm{kHz}$ when the excitation point is at $[0,7.5,0] \mathrm{m}$ in a fixed frame. The excitation force moves from left to right.

For excitation at $500 \mathrm{~Hz}$ (Fig. 12(a)), the sound field is similar to that generated by a compact source with a strong directivity, contributing mainly to observed in the SPL distribution shown in Fig. 12(c). At this frequency, the rail is in the 'blocked region' (see Fig. 11), where both the structural waves decay rapidly with distance. The pass-by spectrum at the reference microphone is also given in Fig. 12(e) This is calculated for a time window corresponding to $26 \mathrm{~m}$.

For excitation at $500 \mathrm{~Hz}$, the sound is captured mostly during the short passage of the force in front of the reference microphone. Due to the Doppler effect, the motion of the source results in a broad peak centred at $500 \mathrm{~Hz}$.

For excitation at $1 \mathrm{kHz}$ (Fig. 12(b)), the sound field is produced by both the structural evanescent and propagating waves since the rail is in the propagating region. The sound field can be separated into three regions, which are identified approximately in the figure by the white lines. In regions 1 and 3, plane waves can be seen. They are generated predominantly by the propagating structural 


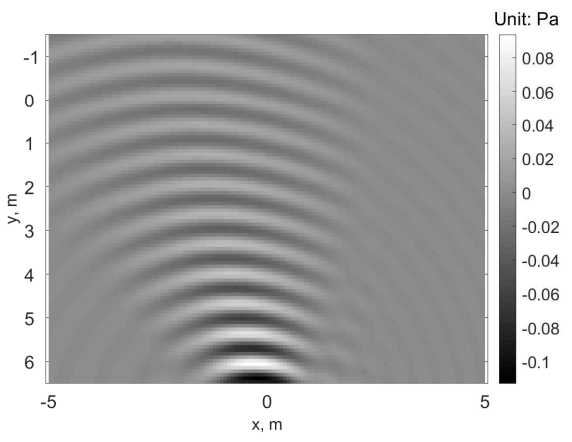

(a)

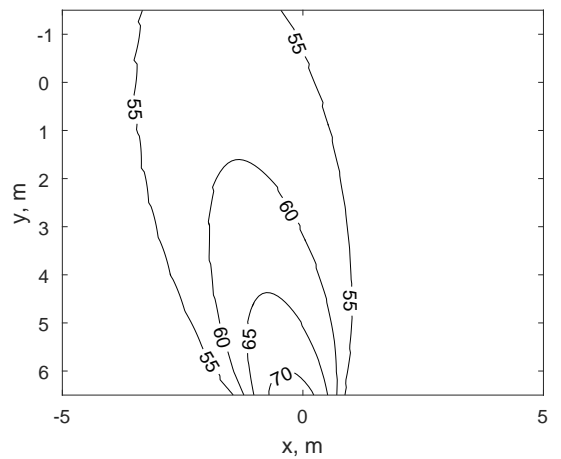

(c)

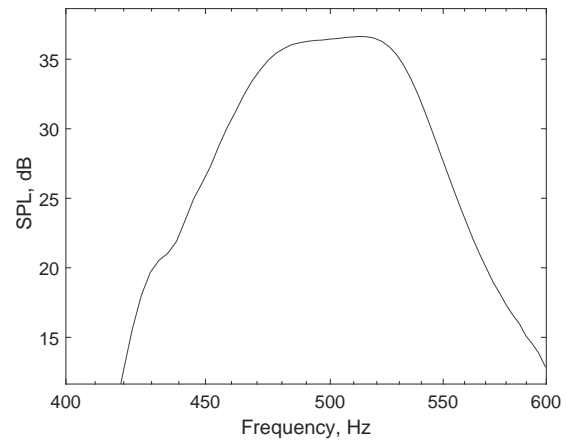

(e)

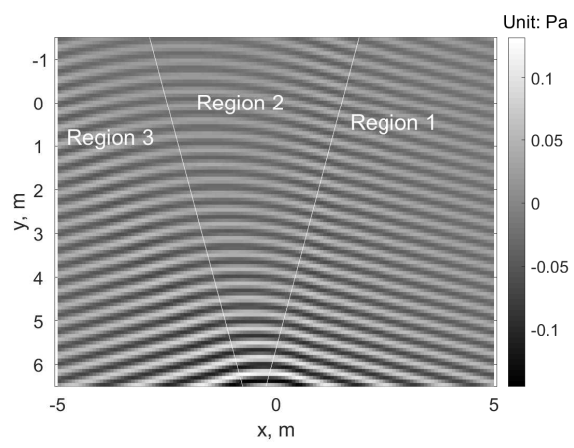

(b)

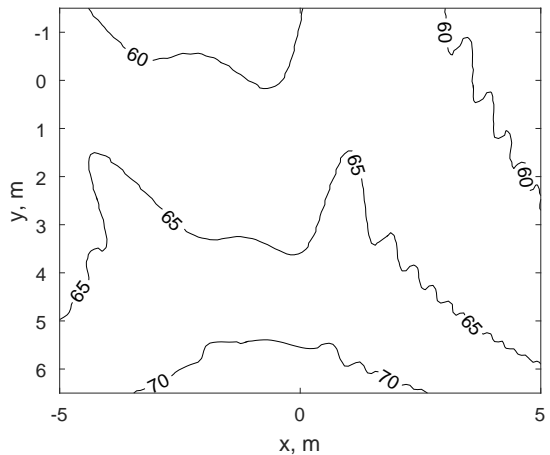

(d)

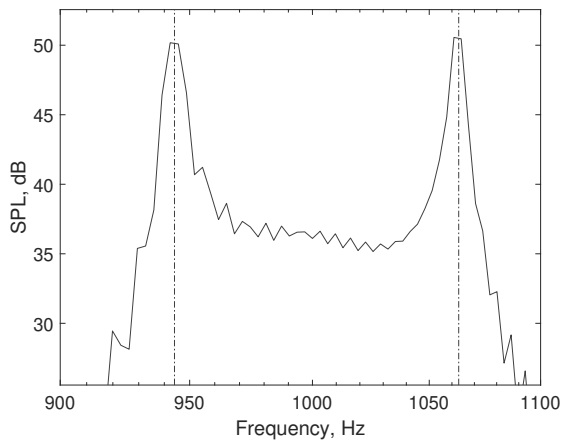

(f)

Figure 12: Rail radiation using a moving excitation at $300 \mathrm{~km} / \mathrm{h}$ : (a) pressure field at 500 $\mathrm{Hz}$, unit: $\mathrm{Pa}$; (b) pressure field at $1 \mathrm{kHz}$, unit: $\mathrm{Pa}$; (c) SPL distribution at $500 \mathrm{~Hz}$; (d) SPL distribution at $1 \mathrm{kHz}$; (e) pass-by spectrum at $500 \mathrm{~Hz}$; (f) pass-by spectrum at $1 \mathrm{kHz}$. 
waves in the rail. These plane waves are the projections of cylindrical waves on the $x-y$ plane. Similarly to the static-excitation case given in [2], the angle of the plane wave propagation relative to the normal $\theta_{\text {prop }}$ can be determined from the propagating structural wavenumbers and the acoustic wavenumbers. Due to the influence of the motion of the excitation on the wavenumbers, this is given by

$$
\theta_{\text {prop }}=\sin ^{-1}\left[\operatorname{Re}\left(k_{r, p}(\omega)\right) / k(\hat{\omega})\right]
$$

where $\hat{\omega}$ is the frequency of the plane waves which can be determined by solving the argument of

$$
\hat{\omega}=\arg \left\{\hat{\omega} \mid \operatorname{Re}\left[k_{r, p}(\omega)\right]=\operatorname{Re}\left[k_{r, p, \text { static }}(\hat{\omega})\right]\right\}
$$

where $k_{r, p \text {,static }}$ denotes the propagating wavenumber for a static excitation. Eqs. (19) and 20) indicate that the moving excitation will not only influence the angles of the plane waves, but also affect the frequencies of these waves. For example in Fig. 12(b) the propagating angle is $13.94^{\circ}$ for the positive direction, which corresponds to a frequency of $1064 \mathrm{~Hz}$. This is obtained by finding the frequency of the load that would give, in a non-moving-load case, the same structural wavelength obtained in the moving-load case. This can be explained through the following steps:

(1) Calculate the wavenumber at the frequency of interest $k_{r, p}$ from the movingload model.

(2) Calculate the spectrum of the wavenumber from the static-load model around the frequency of interest.

(3) Find the $k_{r, p, s t a t i c}$, which has the same real part as $k_{r, p}$, i.e. $\operatorname{Re}\left[k_{r, p, s t a t i c}\right]=$ $\operatorname{Re}\left[k_{r, p}\right]$ on the spectrum.

(4) The corresponding frequency of this wavenumber $k_{r, p, s t a t i c}$ is the acoustic frequency radiated by the rail with a moving load.

Similarly, the angle becomes $14.14^{\circ}$ for the negative direction and the corre410 sponding frequency is $944 \mathrm{~Hz}$. For a monopole pass-by under the same situation, the received frequency will vary in a range from $788 \mathrm{~Hz}$ to $1212 \mathrm{~Hz}$ according 
to Eqs. (1) and 22), see Fig. 5 This is the result of the different wave speeds travelling in the rail and in air. At $1 \mathrm{kHz}$, the wave speed of the rail is 1329 $\mathrm{m} / \mathrm{s}$ for the positive direction and is $1473 \mathrm{~m} / \mathrm{s}$ for the negative direction, which are both much greater than for sound in air, i.e. $340 \mathrm{~m} / \mathrm{s}$. In region 2 , the sound field behaves similarly to that at $500 \mathrm{~Hz}$. It is the result of the coherent combination of the sound field generated by both structural waves.

Fig. 12(f) shows the normalised pass-by spectrum of the rail radiation for excitation at $1 \mathrm{kHz}$; two peaks with similar levels can be seen. These two peaks correspond to the two frequencies, $944 \mathrm{~Hz}$ and $1064 \mathrm{~Hz}$, of the plane waves in regions 1 and 3. Clearly, the predominant contributors to the rail radiation for a load at $1 \mathrm{kHz}$ are the plane waves.

\subsection{Beamforming responses}

Having obtained the rail noise radiation numerically, the responses observed by the array can be calculated. The same simulation settings as used in Sections 2 and 3 are adopted here, except the noise source which is the rail.

Figs. 13 $(a, b)$ show the $1 / 3$ octave band maps of the rail radiation for moving excitation at $500 \mathrm{~Hz}$ and $1 \mathrm{kHz}$ obtained by using a tracking angle $\theta=10^{\circ}$. The peaks of both maps (marked with crosses) are found at $[0,7.5,0] \mathrm{m}$. For 500 Hz, Fig. 13(a) shows an acoustic map typical of a compact source. As shown in Fig. 12(a) and 12(c), the rail only contains waves that decay rapidly since it is in the 'blocked region' at this frequency. The bright spot on the map is expected to be the sound generated by these rapidly decaying structural waves. At $1 \mathrm{kHz}$, Fig. 13(b) again shows a response similar to a compact source in the

435 map centre, which is caused by the noise in region 2. Additionally, the brighter lobe at the centre is now connected to two elongated source regions, one on each side. These two extended sources represent what the array can capture of the cylindrical waves in regions 1 and 3. Their amplitudes are around 15 to $20 \mathrm{~dB}$ lower than the peak. The much lower levels of these extended sources also imply that the beamforming technique for moving monopoles using a short tracking length is not sensitive to the cylindrical waves, which are the major 
source of the rail noise at this frequency. As studied in [2], the array will include a greater contribution from the plane waves if the tracking angle $\theta$ is enlarged. To demonstrate this, the acoustic maps obtained by using tracking angles of $20^{\circ}$ and $30^{\circ}$ are shown in Fig. 13(c) to (f). For excitation at $500 \mathrm{~Hz}$, the maps are almost identical for different tracking angles, whereas the extended sources found at $1 \mathrm{kHz}$ become more evident with increasing $\theta$. Specifically, at $\theta=30^{\circ}$ the plane wave contributions can be seen up to $10 \mathrm{~m}$ away from the map centre.

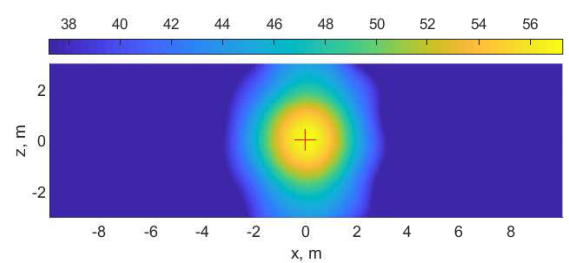

(a)

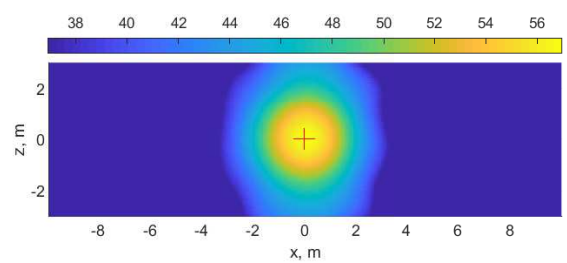

(c)

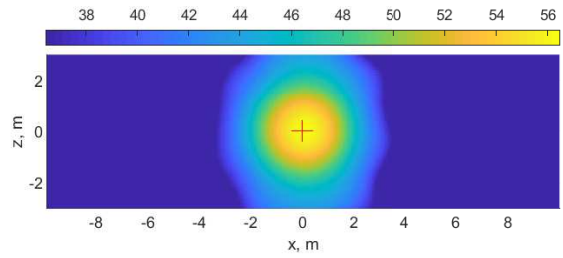

(e)

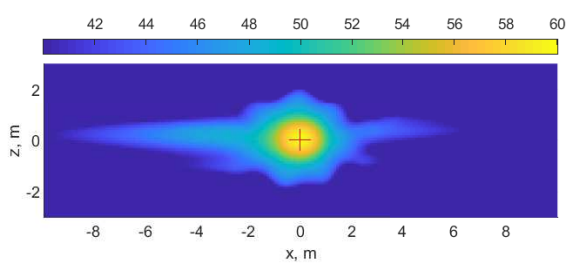

(b)

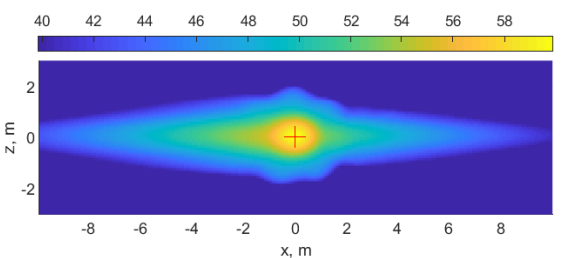

(d)

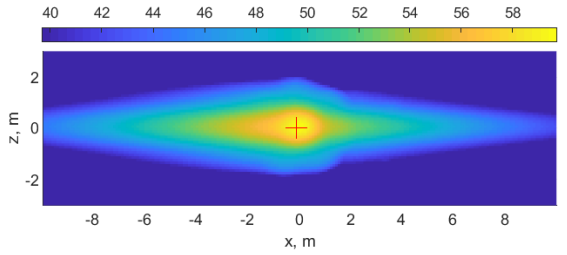

(f)

Figure 13: The $1 / 3$ octave band beamforming maps of rail radiation with moving excitation at $300 \mathrm{~km} / \mathrm{h}$ using different tracking angle $\theta$ : (a) $\theta=10^{\circ}$ at $500 \mathrm{~Hz}$; (b) $\theta=10^{\circ}$ at $1 \mathrm{kHz}$; (c) $\theta=20^{\circ}$ at $500 \mathrm{~Hz}$; (d) $\theta=20^{\circ}$ at $1 \mathrm{kHz}$; (e) $\theta=30^{\circ}$ at $500 \mathrm{~Hz}$; (f) $\theta=30^{\circ}$ at $1 \mathrm{kHz}$. The dynamic ranges of the maps are $20 \mathrm{~dB}$. 


\subsection{Contribution of the rail structural waves}

450 between the structural waves and their contribution to the beamforming maps, an additional numerical analysis is presented in this section. It was shown in Fig. 12 that the rail radiation depends on the structural waves and therefore changes with frequency. The main goal of this analysis is to assess the relative contribution of the sound radiated by the different structural waves on the quantification results. A tracking angle of $\theta=10^{\circ}$ is used throughout this analysis.

\subsubsection{Propagating structural waves}

Firstly, simulations are conducted with a spatial window applied to the source distribution. The shape of the spatial window is shown in Fig. 14 as the solid line. This funnel-shape window with a width of $2 \mathrm{~m}$ is applied to the source strengths obtained from the dynamic model of the rail and moves with the excitation. It reduces the vibration amplitude of the rail in the vicinity of the excitation and ensures that only the propagating waves generate noise. By applying this window, it is expected that the rail noise radiation in region 2 will be reduced while the cylindrical field of regions 1 and 3 is not expected to be significantly influenced.

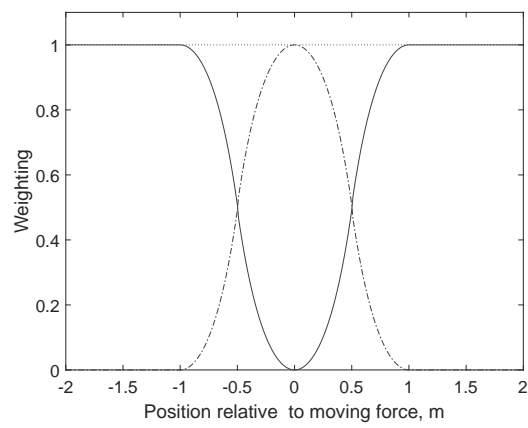

Figure 14: Spatial windows applied for the rail source distribution: '-', window adopted to suppress the rail contribution in the vicinity of the moving excitation. '-.', window adopted to suppress the rail contribution from the propagating waves. 
The maps of the SPL distributions are given in Fig. 15. At $500 \mathrm{~Hz}$, the sound field has three directional lobes, which are labelled from $a$ to $c$. These three lobes have similar SPL distributions and are $15 \mathrm{~dB}$ lower than the original SPL distribution map in Fig. 12(c), In particular, lobes $a$ and $c$ are generated due to the incomplete shading of the windowed rail model, while lobe $b$ is generated due to the coherent superposition of lobes $a$ and $c$. At $1 \mathrm{kHz}$, an acoustic 'vacuum' can be found in the area in front of the excitation, as seen in Fig. 15(b). In the region where the plane waves dominate, the pressures found are similar to those in Fig. 12(d).

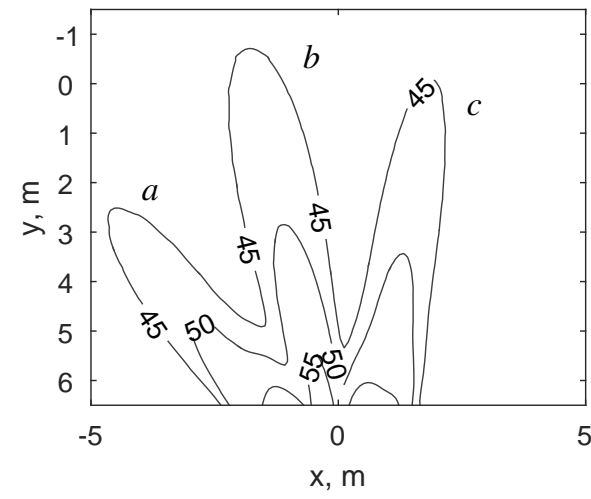

(a)

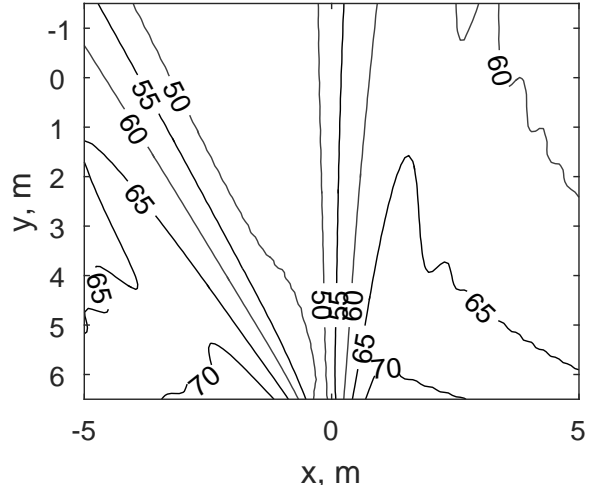

(b)

Figure 15: Windowed SPL distribution maps generated by a moving excitation at $[0,7.5,0] \mathrm{m}$ : (a) at $500 \mathrm{~Hz}$; (b) at $1 \mathrm{kHz}$.

A comparison between the spectra of the reference microphone signal obtained using the model with and without the spatial window is given in Fig. 16. The excitation contains harmonic signals at the central frequencies of the $1 / 3$ octave bands from $500 \mathrm{~Hz}$ to $4 \mathrm{kHz}$. The vertical line denotes the upper frequency limit of the 'blocked region' of this track (see Fig. 11). In the 500 $\mathrm{Hz}$ band, the SPL difference introduced by the windowing is more than $10 \mathrm{~dB}$. Above $500 \mathrm{~Hz}$, the gap between these two spectra reduces since the structural waves in the rail start to propagate. Above $1 \mathrm{kHz}$, the SPL difference is around

$1 \mathrm{~dB}$. This, again, confirms the effectiveness of the window in suppressing the 
noise generated from the vicinity of the excitation. Moreover, this also shows that the predominant contributor to the rail noise is the propagating structural waves when the frequency of interest is above the 'blocked region', whereas it is the region close to the excitation point in the 'blocked region'.

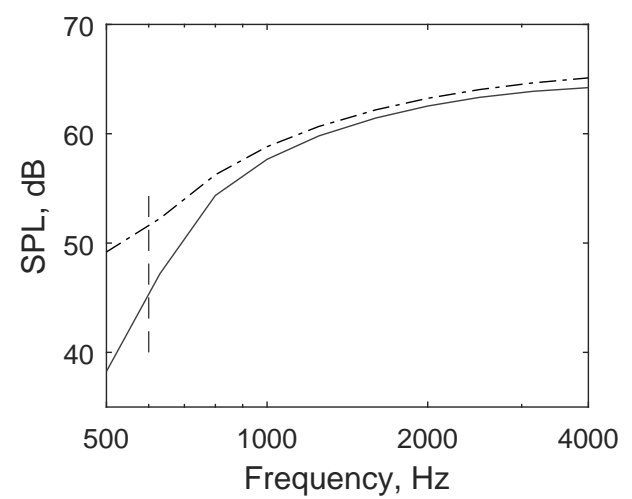

Figure 16: Spectra of the rail radiation with moving excitation: '-', windowed rail model; '-.', the original rail model. The vertical dashed line denotes the upper frequency limit of the 'blocked region'.

The windowed beamforming results are shown in Fig. 17. An identical dynamic range as that used for the original map is adopted for each map to highlight the difference. In Fig. 17(a) at $500 \mathrm{~Hz}$, a compact source with a peak of $44 \mathrm{~dB}$ is found at the centre of the map, which is more than $11 \mathrm{~dB}$ lower than the non-windowed result. In Fig. 17(b) at $1 \mathrm{kHz}$, the bright spot at the map centre disappears. The extended sources, on the other hand, remain similar to those found in Fig. 13(b)

Figs. 15 to 17 verify the relationship between the structural waves and their beamforming responses stated previously. For the rail, the conventional beamforming technique used for railway noise identification mainly captures the con500 tributions coming from the noise radiated in the vicinity of the excitation at all frequencies. When the frequency of interest is above the 'blocked region', although the cylindrical waves are the predominant sources in a single microphone spectrum, they are largely suppressed in the beamforming maps for $\theta=10^{\circ}$. 


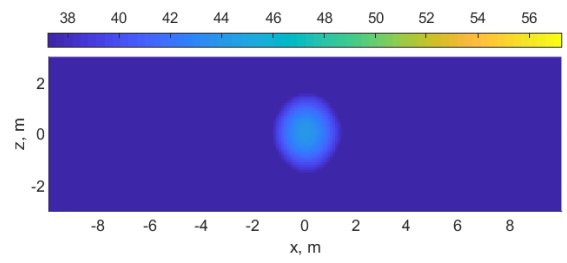

(a)

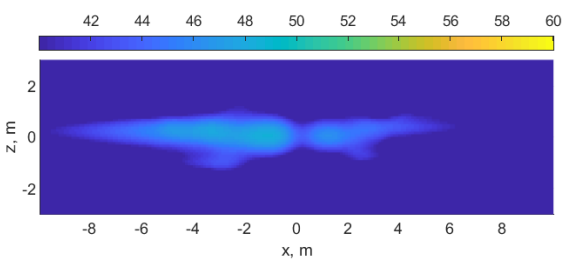

(b)

Figure 17: Beamforming maps of windowed rail radiation with moving excitation: (a) at 500 $\mathrm{Hz}$; (b) at $1 \mathrm{kHz}$.

This demonstrates that the contribution of the propagating waves (away from the forcing point) is negligible in the beamforming maps. A quantification algorithm cannot therefore be applied to this component of the rail radiation.

\subsubsection{Structural waves in the vicinity of the forcing point}

The quantification algorithm introduced in Section 2.2 is here applied to the acoustic maps shown in Fig. 13 to quantify the contribution of the structural waves in the vicinity of the forcing point. The estimated spectrum of the rail radiation based on its beamforming responses is given by a dashed line in Fig. 18 The spectrum of the reference microphone corresponding to a pass-by over $26 \mathrm{~m}$ is given as a dash-dot line. Above $1 \mathrm{kHz}$, the beamforming estimation is slightly lower than the single microphone result, but at $500 \mathrm{~Hz}$ it is $5 \mathrm{~dB}$ greater than 515 it.

The acoustic maps of the rail mainly identify the noise radiated from the vicinity of the excitation, which is highly directional, while they are not influenced by the noise radiated from the propagating structural waves. Therefore, the quantification results from the maps overestimate the noise contributions due to this highly directional noise source, in the region where the cylindrical waves generated by propagating waves are not evident. This is similar to the results shown previously for the simple dipole and quadrupole sources (Fig. 8). Above $1 \mathrm{kHz}$, the overestimation of the noise in the vicinity of the excitation remains. As a consequence, this reduces the difference between the beamform- 


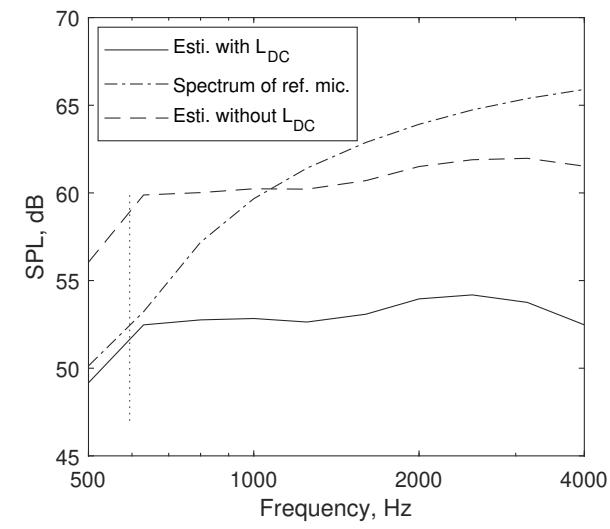

Figure 18: Rail quantification: '-', the beamforming estimation with the directivity compensation; '-.', the single microphone spectrum. '--', the original beamforming estimation. The vertical line shows the upper frequency limit of the 'blocked region'.

As a numerical exercise, the correct beamforming quantification can be found by calculating an additional compensation (see Eq. (8)) based on the directivity of the compact source, since the noise radiated from the vicinity of the excitation of the rail behaves as a highly directional one. $\tilde{p}_{\text {direct }}$ in this case represents the normalised sound pressure signal produced in the vicinity of the excitation. In this case, this cannot be written in an analytical form. Hence, it should be 
obtained through numerical simulation.

To isolate the sound field produced in the vicinity of the excitation, another spatial window, which is the inverse of the one used in Fig. 15, is adopted and is shown in Fig. 14 as a dash-dot line. Moreover, the width of this window, intended to remove the cylindrical waves, is chosen to be frequency dependent. At higher frequency, a smaller window width is used. For instance, the window width is $1.75 \mathrm{~m}$ at $630 \mathrm{~Hz}$ and it becomes $0.53 \mathrm{~m}$ when the frequency of interest is $4 \mathrm{kHz}$. The width of the window is defined by fitting the windowed sound field to the non-windowed one in the area that is mainly associated with the vicinity of the excitation. The SPLs in this area are expected to be similar for the windowed and the non-windowed results. The SPL distributions of the windowed rail are given in Fig. 19. To show the effectiveness of the window, the SPL distributions of the rail without windowing are also shown. Good agreement in terms of SPL can be found in the figure for the areas corresponding to the middle of region 2 , whereas in regions 1 and 3 the noise produced by the propagating waves are greatly suppressed by applying the window.

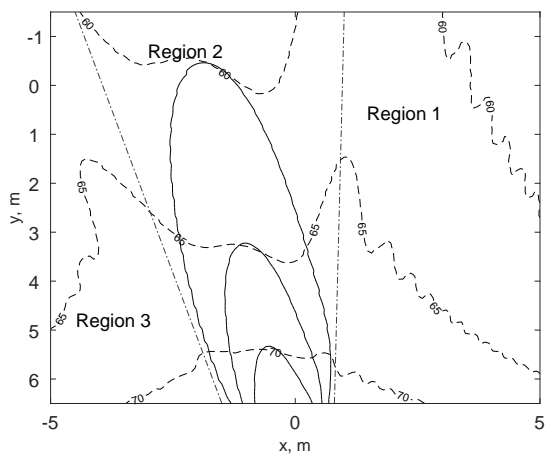

(a)

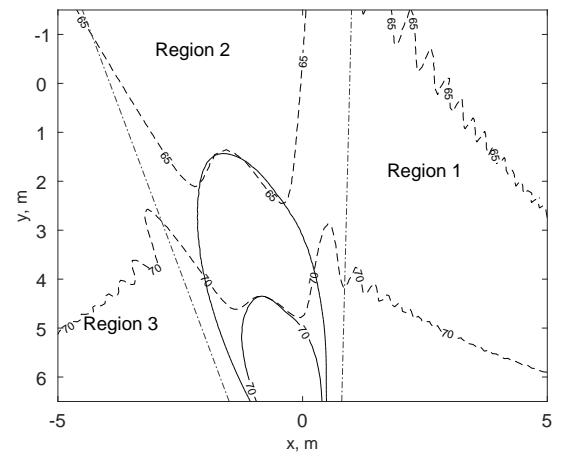

(b)

Figure 19: SPL distribution maps of the rail generated by a moving excitation: (a), at $1 \mathrm{kHz}$; (b), at $2 \mathrm{kHz}$. '-', with the spatial window; '--', without the spatial window. The maps are divided by dash-dot lines to highlight approximately the regions with different characteristics.

By applying the additional compensation based on the directivity of the 
rail, the beamforming estimation is expected to be recovered accurately. This is given in Fig. 18 as a solid line. Unlike the original estimation, a good match can be found between the microphone signal and the beamforming result with the directivity compensation when the excitation is below $630 \mathrm{~Hz}$. Moreover, the overestimation of the original result across the spectrum disappears. The difference between the single microphone result and the revised beamforming result increases with increasing frequency above $630 \mathrm{~Hz}$. The difference is around $8 \mathrm{~dB}$ at $4 \mathrm{kHz}$. The cylindrical sound field is the dominant component of the rail radiation when the frequency of interest is above the 'blocked region', but it is suppressed by the proposed quantification algorithm.

An additional example is given in Fig. 20. In this case, the rail pad stiffness is increased to $2 \times 10^{9} \mathrm{~N} / \mathrm{m}^{2}$, which causes the upper limit of the 'blocked region' to increase to around $1050 \mathrm{~Hz}$. Good matching appears when the frequency is below $1000 \mathrm{~Hz}$, while a level difference appears when the waves start to propagate.

57 In principle, the beamforming technique can be used to identify the noise radiated from the vicinity of the excitation by knowing the sound directivity from this area. However, this can only be applied in a theoretical case when only the rail noise is present. In field tests, the rail contribution is very close to the wheel and these two sources radiate with different directivities. A unique compensation factor cannot be found and beamforming results from field tests include a possible overestimation of the wheel contribution due to the high directivity of the component of rail noise captured by the array.

\section{Conclusions}

A quantification algorithm for railway noise sources has been introduced based on source power integration. It makes use of the beamforming maps in one-third octave bands, evaluated by taking the source motion into account, and can calculate the noise spectra corresponding to the various sources identified on the beamforming maps. 


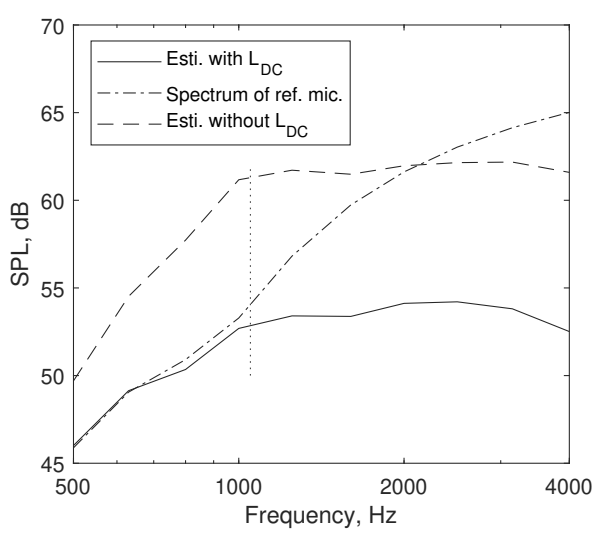

Figure 20: Rail quantification: '-', the beamforming estimation with the directivity compensation; '-.', the single microphone spectrum. '--', the original beamforming estimation. The vertical lines, upper frequency limit of the 'blocked region'. The rail model using a pad stiffness of $2 \times 10^{9} \mathrm{~N} / \mathrm{m}^{2}$.

Numerical simulations have first been used to analyse sources characterised by simple directivities. These are a monopole, a dipole and a longitudinal quadrupole oriented laterally in a direction perpendicular to the direction of motion. Such sources can represent wheel noise and aerodynamic noise. It has been shown that the beamforming-based quantification algorithm can recover the correct spectrum at a reference microphone only if a compensation factor, based on the source directivity, is included in the procedure. For a dipole source, and for a typical railway measurement set-up, the compensation is calculated to be $2.0 \mathrm{~dB}$, whereas it is $3.5 \mathrm{~dB}$ for a quadrupole source.

In addition, an extensive study of the sound field generated by the rail excited by a harmonic moving load has been presented. This is shown to be a combination of the sound radiated by the evanescent and propagating structural waves. It is demonstrated that the classic beamforming approach adopted in the paper does not capture the contribution from the propagating structural waves. Instead, the procedure is strongly influenced by the sound field generated by the rail vibration in the vicinity of the forcing point. This is shown to be a highly directional compact source that can only be quantified correctly if its directivity 
is known. This is not accounted for in previous literature. Additionally, in field tests, the sound field is complicated further by the presence of the wheel that radiates according to a different directivity pattern. As a result, rail and wheel noise cannot be easily separated and part of the rail contribution may be overestimated and incorrectly attributed to the wheel.

\section{Appendix A. Derivation of conversion from beamforming source strength estimation to SPL}

The acoustic field generated by a single monopole source is given by

$$
p(r, t)=\frac{A e^{\mathrm{i} \omega\left(t-\frac{r}{c_{0}}\right)}}{r}
$$

where $r$ is the distance from the source to a receiver, $t$ is the receiving time, $\omega$ denotes the frequency of the source, $c_{0}$ is the speed of sound, and $A$ denotes the amplitude of the source. The power of the source $W$ can be written in terms of the amplitude of the source $A$, which is

$$
W=\frac{2 \pi|A|^{2}}{\rho_{0} c_{0}}
$$

where $\rho_{0}$ is the air density and $c_{0}$ is the sound speed.

The SPL can consequently be obtained as:

$$
L_{p}=10 \log _{10}\left(p_{\mathrm{rms}}^{2} / p_{\mathrm{ref}}^{2}\right)=10 \log _{10}\left(\frac{W}{4 \pi r_{\mathrm{ref}}^{2}} \frac{\rho_{0} c_{0}}{p_{\mathrm{ref}^{2}}}\right)
$$

where $p_{\text {ref }}$ is the reference pressure of $2 \times 10^{-5} \mathrm{~Pa}$.

615 of $(4 \pi|A|)^{2}$. Hence by replacing $|A|^{2}$ with $\tilde{Q}_{j}^{2} / 16 \pi^{2}$, the acoustic map level in terms of SPL yields

$$
L_{p}=10 \log _{10}\left[\widetilde{Q}_{j}^{2}(\omega)\right]+10 \log _{10}\left(\frac{1}{8 \pi p_{\text {ref }}^{2}}\right)-10 \log _{10}\left(4 \pi r^{2}\right)
$$

Since the beamforming result $\widetilde{Q}_{j}^{2}$ is a summation of weighted microphone signals, the distances from the source to the array microphones are different. Therefore, 620 a reference distance is applied to normalise the beamforming estimation. In this paper, it is the distance from the grid $j$ to the centre of the array. 


\section{Appendix B. Derivation of sound radiated by a moving quadrupole}

The sound pressure at $m$-th microphone generated by a moving quadrupole satisfies the wave equation [5, 34]

$$
\left[\frac{1}{c_{0}} \frac{\partial^{2}}{\partial t^{2}}-\nabla^{2}\right] p_{m}(t)=-\frac{\partial^{2}}{\partial y^{2}}\left[T \delta\left(\mathbf{r}_{s}-\mathbf{r}_{m}\right) \delta(t-\tau)\right]
$$

where $\mathbf{r}_{s}$ and $\mathbf{r}_{m}$ denote the position vector of the source and of the $m$-th array microphone, $T$ denotes the source strength of the quadrupole, and, here, $T$ is

described as $\left[0, T_{y} e^{\mathrm{i} \omega \tau}, 0\right]$, which is harmonic with constant amplitude $T_{y}$ in the $y$ direction, for simplicity.

$p_{m}(t)$ in Eq. B.1 has the solution

$$
p_{m}(t)=-\frac{\partial^{2}}{\partial y^{2}}\left(\frac{T}{4 \pi r_{m}(\tau) D\left[r_{m}(\tau)\right]}\right)
$$

The first derivative of Eq. B.2 yields (see Ref. 30 for example)

$$
p_{m}(t)=\frac{\partial}{\partial y}(\underbrace{\frac{\mathrm{i} k T_{y} e^{\mathrm{i} \omega \tau} \cos \phi(\tau)}{4 \pi r_{m}(\tau) D^{2}\left[r_{m}(\tau)\right]}}_{\mathrm{T}_{1}}+\underbrace{\frac{\left(1-M^{2}\right) T_{y} e^{\mathrm{i} \omega \tau} \cos \phi(\tau)}{4 \pi r_{m}^{2}(\tau) D^{3}\left[r_{m}(\tau)\right]}}_{\mathrm{T}_{2}})
$$

where the angle $\phi(\tau)$ is determined by the relative position between the moving source and the receiver. The summation of terms $T_{1}$ and $T_{2}$ in Eq. B.3 is in fact the sound radiation produced by a moving dipole [23, 30]. For the cases studied in this paper where the receiver is placed far from the moving source, $\mathrm{T}_{1}$ is considered to be the major contributor to the dipole sound radiation. Therefore, the sound radiation of a moving dipole can be simplified as

$$
p_{m}(t)=\frac{Q(\tau) \cos \phi(\tau)}{4 \pi r_{m}(\tau) D^{2}\left[r_{m}(\tau)\right]}
$$

where $Q(\tau)=\mathrm{i} k T_{y} e^{\mathrm{i} \omega \tau}$ denotes the generic source strength of the dipole.

By taking the second derivative, the sound radiation generated by a moving quadrupole can be obtained. Specifically,

$$
\begin{aligned}
\frac{\partial}{\partial y} \mathrm{~T}_{1} & =\frac{\mathrm{i} k T_{y} e^{\mathrm{i} \omega \tau}}{4 \pi r_{m}^{2} D^{2}\left[r_{m}\right]}+\frac{(\mathrm{i} k)^{2} T_{y} e^{\mathrm{i} \omega \tau} \cos ^{2} \phi}{4 \pi r_{m} D^{3}\left[r_{m}\right]}-\frac{2 \mathrm{i} k\left(1-M^{2}\right) T_{y} e^{\mathrm{i} \omega \tau} \cos ^{2} \phi}{4 \pi r_{m}^{2} D^{4}\left[r_{m}\right]} \\
\frac{\partial}{\partial y} \mathrm{~T}_{2} & =\left(1-M^{2}\right)\left(\frac{T_{y} e^{\mathrm{i} \omega \tau} \cos ^{2} \phi}{4 \pi r_{m}^{3} D^{3}\left[r_{m}\right]}-\frac{\mathrm{i} k T_{y} e^{\mathrm{i} \omega \tau} \cos ^{2} \phi}{4 \pi r_{m}^{2} D^{4}\left[r_{m}\right]}-\frac{3 T_{y} e^{\mathrm{i} \omega \tau} \cos ^{2} \phi}{4 \pi r_{m}^{3} D^{5}\left[r_{m}\right]}\right)
\end{aligned}
$$


With some algebra, the sound radiation of a moving quadrupole can be written as:

$$
\begin{gathered}
p_{m}(t)=\frac{-k^{2} T_{y} e^{\mathrm{i} \omega \tau}}{4 \pi r_{m} D^{3}\left[r_{m}\right]}\left\{\cos ^{2} \phi[1-\right. \\
\left.-\frac{3\left(1-M^{2}\right)}{\mathrm{i} k r_{m} D\left[r_{m}\right]}-\frac{3\left(1-M^{2}\right)^{2}}{\left(\mathrm{i} k r_{m}\right)^{2} D^{2}\left[r_{m}\right]}\right] \ldots \\
\left.+\frac{D\left[r_{m}\right]}{\mathrm{i} k r_{m}}+\frac{1-M^{2}}{\left(\mathrm{i} k r_{m}\right)^{2}}\right\}
\end{gathered}
$$

For $k r_{m} \gg 1$ and $M \ll 1$, Eq. B.6 can be approximated as

$$
p_{m}(t)=\frac{Q(\tau) \cos ^{2} \phi(\tau)}{4 \pi r_{m}(\tau) D^{3}\left[r_{m}(\tau)\right]}
$$

where $Q(\tau)=-k^{2} T_{y} e^{\mathrm{i} \omega \tau}$ denotes the generic source strength of the quadrupole.

\section{Appendix C. Derivation of Timoshenko beam model of track with moving load}

The derivation shown in this section mainly follows the methods described in 31, 32.

The vibration of a Timoshenko beam excited by a moving harmonic load at frequency $\omega$ can be described by the following differential equations

$$
\begin{aligned}
& G A \kappa \frac{\partial}{\partial x}\left(\phi-\frac{\partial u}{\partial x}\right)+s u+\rho A \frac{\partial^{2} u}{\partial t^{2}}=F e^{\mathrm{i} \omega t} \delta(x-V t) \\
& G A \kappa\left(\phi-\frac{\partial u}{\partial x}\right)-E I \frac{\partial^{2} \phi}{\partial x^{2}}+\rho I \frac{\partial^{2} \phi}{\partial t^{2}}=0
\end{aligned}
$$

where $u$ is the displacement of the beam, $\phi$ denotes the rotation of the crosssection relative to the undeformed axis, and $V$ is the velocity of the excitation point. Other parameters are defined in Table 1. The argument of the Dirac function in Eq. C.1 describes the motion of the excitation.

The rail displacement $u$ can be found by transforming Eq. (C.1) and Eq. (C.2) from the space-time domain $(x, t)$ to the wavenumber-frequency domain $(k, \widetilde{\omega})$. These two Fourier transform pairs are given by

$$
\begin{aligned}
& U(\widetilde{\omega})=\int_{-\infty}^{\infty} u(t) e^{-\mathrm{i} \widetilde{\omega} t} d t ; u(t)=\frac{1}{2 \pi} \int_{-\infty}^{\infty} U(\widetilde{\omega}) e^{\mathrm{i} \widetilde{\omega} t} d \widetilde{\omega} \\
& U(k)=\int_{-\infty}^{\infty} u(x) e^{\mathrm{i} k x} d t ; u(x)=\frac{1}{2 \pi} \int_{-\infty}^{\infty} U(k) e^{-\mathrm{i} k x} d k
\end{aligned}
$$


Hence, applying Eqs. (C.3) and (C.4 to Eqs. C.1 and (C.2) gives

$$
\begin{aligned}
& -G A \kappa \mathrm{i} k(\Phi+\mathrm{i} k U)+s U-\rho A \widetilde{\omega}^{2} U=2 \pi F \delta(\widetilde{\omega}-\omega-k V) \\
& G A \kappa(\Phi+\mathrm{i} k U)+E I k^{2} \Phi-\rho I \widetilde{\omega}^{2} \Phi=0
\end{aligned}
$$

Therefore, the rail displacement $U(k, \omega)$ can be obtained as

$$
U(k, \widetilde{\omega})=\frac{f_{2}(k, \widetilde{\omega})}{f_{1}(k, \widetilde{\omega})} 2 \pi F \delta(\widetilde{\omega}-\omega-k V)
$$

where

$$
\begin{aligned}
& f_{1}(k, \widetilde{\omega})=(G A \kappa k)^{2}-\left(G A \kappa+E I k^{2}-\rho I \widetilde{\omega}^{2}\right)\left(G A \kappa k^{2}+s-\rho A \widetilde{\omega}^{2}\right) \\
& f_{2}(k, \widetilde{\omega})=-G A \kappa-E I k^{2}+\rho I \widetilde{\omega}^{2}
\end{aligned}
$$

Firstly, transforming $U(k, \widetilde{\omega})$ to the wavenumber-time domain by applying the inverse Fourier transform to Eq. C.7), gives

$$
U(k, t)=\frac{f_{2}(k, \widetilde{\omega}=\omega+k V)}{f_{1}(k, \widetilde{\omega}=\omega+k V)} F e^{\mathrm{i}(\omega t+k V t)}
$$

Secondly, to obtain the signal in the space-time domain, another inverse Fourier transform is required. Therefore, the rail displacement $u(x, t)$ is given by

$$
u(x, t)=\frac{F e^{\mathrm{i} \omega t}}{2 \pi} \int_{-\infty}^{\infty} \frac{f_{2}(k, \widetilde{\omega}=\omega+k V)}{f_{1}(k, \widetilde{\omega}=\omega+k V)} e^{-\mathrm{i} k(x-V t)} d k
$$

Eq. C.11 can be evaluated using contour integration. The expression for the rail displacement $u(x, t)$ is given by

$$
\begin{aligned}
& u^{+}(x, t)=-\mathrm{i} F e^{\mathrm{i} \omega t} \sum_{k_{r}^{+}=k_{r, p}^{+}, k_{r, n}^{+}}\left[\frac{p_{n}\left(k_{r}^{+}\right)}{p_{d}\left(k_{r}^{+}\right)} e^{-\mathrm{i} k_{r}^{+}(x-V t)}\right] \\
& x \geqslant V t, \operatorname{Im}\left(k_{r}^{+}\right)<0 \\
& u^{-}(x, t)=\mathrm{i} F e^{\mathrm{i} \omega t} \sum_{k_{r}^{-}=k_{r, p}^{-}, k_{r, n}^{-}}\left[\frac{p_{n}\left(k_{r}^{-}\right)}{p_{d}\left(k_{r}^{-}\right)} e^{-\mathrm{i} k_{r}^{-}(x-V t)}\right] \\
& x \leqslant V t, \operatorname{Im}\left(k_{r}^{-}\right)>0
\end{aligned}
$$

where $p_{n}(k)$ and $p_{d}(k)$ are given by

$$
\begin{aligned}
& p_{n}(k)=C_{n 2} k^{2}+C_{n 1} k+C_{n 0} \\
& p_{d}(k)=4 C_{d 4} k^{3}+3 C_{d 3} k^{2}+2 C_{d 2} k+C_{d 1}
\end{aligned}
$$


Table C.2: Definition of the coefficients in Eq. C.14 and Eq. C.15

\begin{tabular}{ll}
\hline Coefficient & Definitions \\
\hline$C_{n 2}$ & $\rho I V^{2}-E I$ \\
$C_{n 1}$ & $2 \rho I V \omega$ \\
$C_{n 0}$ & $\rho I \omega-G A \kappa$ \\
$C_{d 4}$ & $-E I G A \kappa+E I \rho A V^{2}+\rho I G A \kappa V^{2}-\rho^{2} A I V^{4}$ \\
$C_{d 3}$ & $\left(-4 \rho^{2} A I V^{3}+2 \rho I G A \kappa V+2 E I \rho A V\right) \omega$ \\
$C_{d 2}$ & $\left(\rho I G A \kappa+E I \rho A-6 \rho^{2} A I V^{2}\right) \omega^{2}-E I s+\rho G A^{2} \kappa V^{2}+\rho I s V^{2}$ \\
$C_{d 1}$ & $-4 \rho^{2} A I V \omega^{3}+\left(2 \rho G A^{2} \kappa V+2 \rho I s V\right) \omega$ \\
$C_{d 0}$ & $-\rho^{2} A I \omega^{4}+\left(\rho G A^{2} \kappa+\rho I s\right) \omega^{2}-G A \kappa s$ \\
\hline
\end{tabular}

The wavenumbers $k_{r}$ in the above equation are the poles of the integrand and are obtained by solving

$$
C_{d 4} k^{4}+C_{d 3} k^{3}+C_{d 2} k^{2}+C_{d 1} k+C_{d 0}=0
$$

The definitions of the coefficients that appear in Eqs. (C.14, C.15) and C.16) are given in Table C.2.

It is worth mention that the derivations presented here give the rail response in a fixed space frame rather than one that moves with the excitation. According to 31, the angular frequency $\hat{\omega}$ observed by one given point on the rail is given by

$$
\hat{\omega}=\omega\left(1+V k_{r} / \omega\right)=\omega\left(1+V / c_{\text {rail }}\right)
$$

where $c_{\text {rail }}$ denotes the propagation velocity of the structural wave. Eq. C.17) also explains the Doppler effect caused by the moving excitation.

The point velocity $v$ can be obtained by taking the derivative of the deformation $u$ in Eqs. C.12 and C.13 in terms of time $t$, which is given by 


$$
\begin{aligned}
& v^{+}(x, t)=\frac{\partial u^{+}}{\partial t}=F \sum_{k_{r}^{+}}\left[\frac{p_{n}\left(k_{r}^{+}\right)}{p_{d}\left(k_{r}^{+}\right)}\left(\omega+k_{r}^{+} V\right) e^{\mathrm{i}\left(\omega+k_{r}^{+} V\right) t-\mathrm{i} k_{r}^{+} x}\right] \\
& x \geqslant V t, \operatorname{Im}\left(k_{r}^{+}\right)<0 \\
& v^{-}(x, t)=\frac{\partial u^{-}}{\partial t}=-F \sum_{k_{r}^{-}}\left[\frac{p_{n}\left(k_{r}^{-}\right)}{p_{d}\left(k_{r}^{-}\right)}\left(\omega+k_{r}^{+} V\right) e^{\mathrm{i}\left(\omega+k_{r}^{-} V\right) t-\mathrm{i} k_{r}^{-} x}\right] \\
& x \leqslant V t, \operatorname{Im}\left(k_{r}^{-}\right)>0
\end{aligned}
$$

where the time-dependent terms in the exponential terms in these equations indicate the Doppler effect caused by the source motion, which is important for the study of the rail noise radiation. The magnitude term, on the other hand, generated by the differentiation, can be neglected for the simulations presented in this paper. This is because the speed of the excitation (around $83 \mathrm{~m} / \mathrm{s}$ ) is much smaller compared to the speed of the structural waves (between $1200 \mathrm{~m} / \mathrm{s}$ to $1900 \mathrm{~m} / \mathrm{s}$ ), and, therefore, $\hat{\omega} \approx \omega$.

[1] D. Thompson, Railway noise and vibration: mechanisms, modelling and means of control, Elsevier, 2008.

[2] T. Kitagawa, D. J. Thompson, The horizontal directivity of noise radiated by a rail and implications for the use of microphone arrays, Journal of sound and vibration 329 (2) (2010) 202-220.

[3] D. J. Thompson, E. Latorre Iglesias, X. Liu, J. Zhu, Z. Hu, Recent developments in the prediction and control of aerodynamic noise from high-speed trains, International Journal of Rail Transportation 3 (3) (2015) 119-150.

[4] N. Curle, The influence of solid boundaries upon aerodynamic sound, Proc. R. Soc. Lond. A 231 (1187) (1955) 505-514.

[5] M. J. Lighthill, On sound generated aerodynamically i. general theory, Proc. R. Soc. Lond. A 211 (1107) (1952) 564-587. 
[6] D. Thompson, M. Dittrich, Wheel response and radiation-laboratory measurements of five types of wheel and comparisons with theory, ORE Technical Document DT248 (C163) Utrecht (June 1991).

[7] D. Thompson, B. Hemsworth, N. Vincent, Experimental validation of the TWINS prediction program for rolling noise, part 1: description of the model and method, Journal of Sound and Vibration 193 (1) (1996) 123135.

[8] D. Thompson, P. Fodiman, H. Mahé, Experimental validation of the TWINS prediction program for rolling noise, part 2: results, Journal of Sound and Vibration 193 (1) (1996) 137-147.

[9] E. Zea, L. Manzari, G. Squicciarini, L. Feng, D. Thompson, I. Lopez Arteaga, Wavenumber-domain separation of rail contribution to pass-by noise, Journal of Sound and Vibration 409 (2017) 24-42.

[10] D. Thompson, G. Squicciarini, J. Zhang, I. Lopez Arteaga, E. Zea, M. Dittrich, E. Jansen, K. Arcas, E. Cierco, F. X. Magrans, et al., Assessment of measurement-based methods for separating wheel and track contributions to railway rolling noise, Applied Acoustics 140 (2018) 48-62.

[11] B. Barsikow, W. King III, On removing the Doppler frequency shift from array measurements of railway noise, Journal of Sound and Vibration 120 (1) (1988) 190-196.

[12] P. Nelson, chapter titled 'Source identification and location', in Advanced Applications in Acoustics, Noise and Vibration, F. Fahy and J. Walker, CRC Press, 2004.

[13] B. Barsikow, Experiences with various configurations of microphone arrays used to locate sound sources on railway trains operated by the DB AG, Journal of Sound and Vibration 193 (1) (1996) 283-293. 
[14] H.-M. Noh, S. Choi, S. Hong, S.-W. Kim, Investigation of noise sources in high-speed trains, Proceedings of the Institution of Mechanical Engineers, Part F: Journal of Rail and Rapid Transit 228 (3) (2014) 307-322.

[15] B. Barsikow, W. King III, E. Pfizenmaier, Wheel/rail noise generated by a high-speed train investigated with a line array of microphones, Journal of Sound and Vibration 118 (1) (1987) 99-122.

[16] M. Dittrich, M. Janssens, Improved measurement methods for railway rolling noise, Journal of Sound and Vibration 231 (3) (2000) 595-609.

[17] F. Poisson, P. Gautier, F. Letourneaux, Noise sources for high speed trains: a review of results in the TGV case, in: Noise and vibration mitigation for rail transportation systems, NNFM, Vol. 99, 2008, pp. 71-77.

[18] G. Howell, A. Bradley, M. McCormick, J. Brown, De-dopplerization and acoustic imaging of aircraft flyover noise measurements, Journal of Sound and Vibration 105 (1) (1986) 151-167.

[19] F. Le Courtois, J.-H. Thomas, F. Poisson, J.-C. Pascal, Genetic optimisation of a plane array geometry for beamforming. application to source localisation in a high speed train, Journal of Sound and Vibration 371 (2016) 78-93.

[20] S. Brühl, A. Röder, Acoustic noise source modelling based on microphone array measurements, Journal of Sound and Vibration 231 (3) (2000) 611617.

[21] B. He, X.-b. Xiao, Q. Zhou, Z.-h. Li, X.-s. Jin, Investigation into external noise of a high-speed train at different speeds, Journal of Zhejiang University SCIENCE A 15 (12) (2014) 1019-1033.

[22] F. Le Courtois, J. Thomas, F. Poisson, J. Pascal, Méthode de déconvolution pour l'imagerie des sources en mouvement rapide, in: CFA Poitiers, 2014, p. 1075. 
[23] P. M. Morse, K. U. Ingard, Theoretical acoustics, Princeton university press, 1986.

[24] D. G. Crighton, A. P. Dowling, J. Ffowcs-Williams, M. Heckl, F. Leppington, J. F. Bartram, Modern methods in analytical acoustics lecture notes (1992).

[25] F.-R. Grosche, H. Stiewitt, B. Binder, Acoustic wind-tunnel measurements with a highly directional microphone, AIAA Journal 15 (11) (1977) 15901596.

[26] C. S. Allen, W. K. Blake, R. P. Dougherty, D. Lynch, P. T. Soderman, J. R. Underbrink, Aeroacoustic measurements, Springer Science \& Business Media, 2013.

[27] T. Brooks, W. Humphreys, Jr, Effect of directional array size on the measurement of airframe noise components, in: 5th AIAA/CEAS Aeroacoustics Conference and Exhibit, 1999, p. 1958.

[28] ISO 3095 Railway applications - acoustics - measurement of noise emitted by railbound vehicles, in: International standardization organisation, 2013.

[29] P. Sijtsma, Experimental techniques for identification and characterisation of noise sources, in: National Aerospace Laboratory NLR, 2004.

[30] M. J. Crocker, Handbook of acoustics, John Wiley \& Sons, 1998.

[31] M. Hussein, H. Hunt, Modelling of floating-slab tracks with continuous slabs under oscillating moving loads, Journal of Sound and Vibration 297 (1-2) (2006) 37-54.

[32] X. Sheng, C. Jones, M. Petyt, Ground vibration generated by a load moving along a railway track, Journal of sound and vibration 228 (1) (1999) 129 156. 
[33] D. Thompson, C. Jones, N. Turner, Investigation into the validity of twodimensional models for sound radiation from waves in rails, The Journal of the Acoustical Society of America 113 (4) (2003) 1965-1974.

[34] Y. Mao, C. Xu, D. Qi, H. Tang, Series expansion solution for sound radiation from rotating quadrupole point source, AIAA journal 52 (5) (2014) $1086-1095$. 